halten beschäftigt. Nachdem die Politische Soziologie in Deutschland hier einen enormen Nachholbedarf hat, stellen sich für die Zukunft vielfältige Fragen: Warum variiert das Wissen (zwischen Themen, aber auch zwischen Bürgern) so stark? Warum kann kein Anwachsen von Kenntnissen beobachtet werden, obwohl sich die politischen, sozialen und technologischen Rahmenbedingungen in den letzten Jahrzehnten dramatisch geändert haben? Durch welche Größen auf der Mikro- (zum Beispiel Sozialstruktur, Einstellungen, Mediennutzungsverhalten, Vorwissen) und der Makroebene (zum Beispiel Informationsumwelten, Systemstrukturen) wird das Politikwissen beeinflusst? Und welche Rolle spielen die bei der Messung zum Einsatz kommenden Erhebungsmethoden? Welche Konsequenzen ergeben sich zum Beispiel für persönliche Überzeugungssysteme, individuelles politisches Verhalten, aber auch für demokratische Systeme, wenn Bürger politische Experten oder politische Novizen sind? Last but not least: Welche Fragen eignen sich eigentlich im Rahmen von Befragungen, um zuverlässig auf das - selbstverständlich viel umfassendere - politische Wissen von Bürgern zu schließen - und was sagen die Ergebnisse solcher Wissenstests darüber aus, was Bürger von Politik tatsächlich verstehen?

\title{
Die Strategie der kommunizierten Ehrlichkeit im CDU/CSU-Bundestagswahlkampf 2005
}

\author{
Volker Best
}

Nach der Neuwahlankündigung durch Bundeskanzler Gerhard Schröder und SPD-Parteichef Franz Müntefering am 22. Mai 2005 musste der folgende Bundestagswahlkampf "gleichsam aus dem Boden gestampft werden"1. Umso bemerkenswerter scheint, dass ausgerechnet dieser Wahlkampf eine Innovation hervorbrachte: die Strategie der kommunizierten Ehrlichkeit von CDU und CSU, die im Folgenden hinsichtlich ihrer Motivation und Ausgestaltung, ihrer Probleme und Effekte analysiert werden soll.

\section{Strategien im Wahlkampf der Union 2005}

Die Ehrlichkeitsstrategie kann nicht isoliert von den anderen Wahlkampfstrategien der Union betrachtet werden. Fünf sind hier aus Sicht des Verfassers zu identifizieren ${ }^{2}$ : Es sollte erstens ein detailliertes Regierungsprogramm vorgelegt werden (gouvernementaler Wahlkampf ${ }^{3}$ ). Zweitens sollte sich der Wahlkampf an Themen statt an Personen orientieren

1 Christina Holtz-Bacha, Bundestagswahl 2005 - Die Überraschungswahl, in: dies. (Hrsg.), Die Massenmedien im Wahlkampf. Die Bundestagswahl 2005, Wiesbaden 2006, S. 5 - 31, S. 5.

2 Wahlkämpfe sind Bestandteil des Arkanbereichs von Politik; daher sind Kampagnenziele und -entscheidungen empirisch nicht einfach zu untersuchen. Vgl. Harald Schoen, Wahlkampfforschung, in: ders. / Jürgen W. Falter (Hrsg.), Handbuch Wahlforschung, Wiesbaden 2005, S. 503 -542, S. 505.

3 Dieser Begriff stammt von Frank Brettschneider, Bundestagswahlkampf und Medienberichterstattung, in: APuZ, B $51 / 52$ (2005), S. 19 - 26, S. 22. 
(Themenwahlkampf). Als dominierendes Thema war dabei drittens die wirtschaftliche Situation des Landes vorgesehen (Wirtschaftswahlkampf). Viertens sollte das Ziel einer Koalitionsregierung zusammen mit der FDP klar postuliert (Lagerwahlkampf) und fünftens zu Gunsten eines einheitlichen Erscheinungsbildes im gesamten Land auf einen speziellen Wahlkampf für die neuen Bundesländer verzichtet werden (gesamtdeutscher Wahlkampf).

\subsection{Gouvernementaler Wahlkampf}

Die übliche Rollenverteilung wurde im Bundestagswahlkampf 2005 ausgesetzt: Eine „reale Regierungsopposition“ stand einer „gefühlte[n] Oppositionsregierung“ gegenüber. ${ }^{4}$ In einem gewissen Maße war die Union angesichts der Erwartungshaltung der Wählerschaft und der Medien gegenüber der demoskopisch designierten Regierungspartei nolens volens zu einem über bloße Kritik am Regierungshandeln hinausweisenden gouvernementalen Wahlkampf gezwungen. ${ }^{5}$ Während Oppositionsparteien ihre Herrschaftsansprüche gern mit einem „Regierungsprogramm“ unterstreichen, ging im Fall von CDU und CSU die große Mehrheit der Wähler davon aus, dass dieses Programm auch tatsächlich umgesetzt werde. ${ }^{6}$

Weil die mühsam errungenen programmatischen Kompromisse der Oppositionsjahre aufgrund ihrer mangelnden Vereinbarkeit und Finanzierbarkeit für einen gouvernementalen Wahlkampf kaum taugten ${ }^{7}$ und notwendige programmatische Klärungsprozesse in der Kürze der Zeit nicht mehr vollzogen werden konnten ${ }^{8}$, wurde die Erstellung des Wahlprogramms an eine kleine, abgeschottete Planungsgruppe delegiert. Viele Unionspolitiker

4 Begriffspaar bei: Stephan Eisel, Reale Regierungsopposition gegen gefühlte Oppositionsregierung. Zur Bundestagswahl 2005, in: Die Politische Meinung, Nr. 12/2005, S. 47 - 52.

5 Vgl. Oskar Niedermayer, Der Wahlkampf zur Bundestagswahl 2005. Parteistrategien und Kampagnenverlauf, in: ders. / Frank Brettschneider / Bernhard Weßels (Hrsg.), Die Bundestagswahl 2005. Analysen des Wahlkampfes und der Wahlergebnisse, Wiesbaden 2007, S. 21 - 42, S. 30.

6 Die SPD nannte ihr Programm hingegen bezeichnenderweise „Wahlmanifest“, woraus ein reduzierter Herrschaftsanspruch herausgelesen werden könnte.

7 Auf dem Leipziger Parteitag im Dezember 2003 waren sowohl die von einer Kommission um Altbundespräsident Roman Herzog entwickelte „Kopfpauschale“ als auch die von Friedrich Merz konzipierte große „Bierdeckel“-Steuerreform mit den drei Stufentarifen 12, 24 und 36 Prozent (bei Streichung eines Großteils der Ausnahmetatbestände) beschlossen worden. Der geplante Sozialausgleich im Kopfprämienmodell hätte nach Schätzung von CDU-Experten allerdings 10 bis 20 Milliarden Euro gekostet. Der saarländische Ministerpräsident Peter Müller hielt daher zur Finanzierung der Gesundheitsprämie Steuererhöhungen für unvermeidlich; vgl. SZ vom 1. Dezember 2003, S. 1. Merz' Steuerkonzept hätte demgegenüber weitere Mindereinnahmen bedeutet. Nach seiner eigenen Auskunft bewegten sich diese zwischen fünf und zehn Milliarden Euro. Nach Berechnungen des Finanzministeriums hätte ein kurzfristig eingefügter Arbeitnehmerfreibetrag weitere fünf Milliarden Euro Ausfälle bedeutet, zudem summierten sich die Mindereinnahmen im ersten Jahr - weil der Wegfall von Steuervergünstigungen erst nach und nach zusätzliche Einnahmen bringe - auf 25 Milliarden Euro; vgl. SZ vom 29. November 2003, S. 19. Die Kopfpauschale wurde danach in einem Kompromiss mit der CSU so verändert, dass das Ergebnis in weiten Teilen der CDU als „Murks“ galt, vgl. Jens Schneider, Radikale Rezepte, vorsichtig dosiert, in: SZ vom 11. Juli 2005, S. 2.

8 Man kann insofern eine „konzeptionelle Überrumpelung“ der Union feststellen, vgl. Hans Jörg Hennecke, Von der ,Agenda 2010' zur ,Agenda Merkel'?, in: APuZ, B 32 / 33 (2005), S. 16 - 22, S. 22. 
suchten allerdings - vielleicht auch ermutigt durch das Postulat eines ehrlichen Wahlkampfs -, ihre Exklusion aus dem Prozess der Programmformulierung durch öffentliches „lautes Nachdenken“ zu kompensieren. Neben diversen sachpolitischen Ankündigungen stellte die Union den Wählern auch einen neuen Regierungsstil in Aussicht: eine „handwerklich saubere“ Politik „aus einem Guss“ auf der Basis klarer Mehrheitsverhältnisse im föderalen Bundesstaat, die es erlauben würden, „einmal zwischen Bundestag und Bundesrat durchzuregieren"?.

Insgesamt hat der gouvernementale Wahlkampf - so alternativlos er gewesen sein mag - CDU und CSU mehr geschadet als genützt. Erstens ermöglichte er es der SPD, ihrerseits einen Oppositionswahlkampf als Abwehrschlacht gegen eine bedrohlich durchregierte und sozial kalte „schwarze Republik“ zu führen, wodurch ihr eigenes Programm und ihre Regierungsbilanz in den Hintergrund traten. Zweitens konnten die Titulierung des Wahlprogramms als „Regierungsprogramm“, die ständigen Spekulationen über Kabinettslisten und die Kommunikation der Umfragezahlen als quasi vorweggenommene Wahlentscheidung als „mangelnde Demut vor dem Wählerwillen“ aufgefasst werden, die Trotzreaktionen „von der SPD umworben[er], von der Union bereits vereinnahmt[er]“, selbstbewusster Wechselwähler hervorrief. ${ }^{10}$ Drittens schwächte die Siegesgewissheit der Union auch ihre Angriffskraft, da sich die Mitglieder des Kompetenzteams eher als zukünftige Regierungsmitglieder denn als aggressive Wahlkämpfer sahen. ${ }^{11}$ Genutzt hat der Union der Regierungswahlkampf insofern, als er erstens ihren Machtanspruch bekräftigte und zweitens ihrer Kandidatin Angela Merkel die Rolle der "gefühlten Kanzlerin"12 zuwies und deren Vermittlung an das Wahlvolk weitgehend obsolet machte, was die Erfolgschancen der zweiten Strategie verbesserte: des Verzichts auf Personalisierung zugunsten eines Themenwahlkampfs.

\subsection{Themenwahlkampf}

Der Union musste an einer umfassenden Entpersonalisierung und Versachlichung des Wahlkampfs gelegen sein, denn die Medien attestierten ihrer Kanzlerkandidatin einhellig eine „eingeschränkte charismatische Wirkung “13. Angesichts Schröders überlegener Persönlichkeitswerte war es für die Union naheliegend, auf den Vorsprung der Partei wie der Spitzenkandidatin an Sachkompetenz in den Augen der Wähler ${ }^{14}$ zu setzen. Hilfreich war für die Union, dass Schröder den Wahlkampf gegen die erste weibliche Kanzlerkandidatin in der Geschichte der Bundesrepublik Deutschland nicht analog zu 2002 gegen Edmund Stoiber auf die Frage "Sie oder ich“ zuspitzen konnte. ${ }^{15}$ Eine Wiederholung der im Wahlkampf 2002 erstmals durchgeführten TV-Duelle lag nicht im Interesse der Union. Gänz-

9 Zitiert nach Welt am Sonntag vom 7. August 2005, S. 4; SZ vom 2. Juli 2005, S. 1; FAZ vom 24. Juni 2005, S. 3.

10 Stephan Eisel, a.a.O. (Fn. 4), S. 50.

11 Vgl. ebenda.

12 Ebenda, S. 49.

13 Matthias Jung / Andrea Wolf, Der Wählerwille erzwingt die große Koalition, in: APuZ, B 51 / 52 (2005), S. 3 - 12, S. 6. Selbst dezidiert konservative Tageszeitungen teilten diese Einschätzung, vgl. zum Beispiel FAZ vom 15. August 2005, S. 33; Die Welt vom 20. August 2005, S. 3.

14 Vgl. Infratest dimap, ARD-DeutschlandTrend, Mai 2005 II, Extra „Neuwahl“, S. 12 f.

15 Vgl. Christina Holtz-Bacha, Personalisiert und emotional. Strategien des modernen Wahlkampfes, in: APuZ, B 7 (2006), S. 11 - 19, S. 17 f. 
lich versagen konnte sich Merkel dieser „bereits durch ihre erste Durchführung zur Institution geworden[en] "16 Form medialer Auseinandersetzung zwar nicht, die Beschränkung auf nur eine Fernseh-Debatte sollte ihr aber einen Achtungserfolg gegen den Medienkanzler ermöglichen. Die Dichotomie der Spitzenkandidaten wurde durch die Präsentation eines Kompetenzteams weiter entschärft.

Auch wenn im Plakatwahlkampf paradoxerweise die SPD die markanteren Aussagen beisteuerte, während die CDU/CSU mit austauschbaren Standard-Slogans warb ${ }^{17}$, war die Strategie der Union, einen Personenwahlkampf zu vermeiden und stattdessen einen Themenwahlkampf zu führen, erfolgreich: Parteipositionen zu Sachfragen spielten 2005 für 58 gegenüber 2002 nur 48 Prozent der Wähler die entscheidende Rolle; nur noch 18 gegenüber vormals 29 Prozent machten ihre Entscheidung am Spitzenkandidaten fest. ${ }^{18}$

\subsection{Wirtschaftswahlkampf}

Damit ist freilich noch keine Aussage über die Auswahl der politischen Vorschläge und Ankündigungen zur Ausfüllung des Themenwahlkampfs gemacht. Die Union entschied sich wie schon 2002 für einen Wirtschaftswahlkampf. Diese Akzentuierung ließ sich bereits dem Titel des Regierungsprogramms „Deutschlands Chancen nutzen. Wachstum. Arbeit. Sicherheit" entnehmen. Fünf Sechstel der Einleitung widmeten sich ausschließlich der Wirtschaft, den Hauptteil dominierte das Kapitel „Vorfahrt für Arbeit“ mit 15 von 30 Seiten. ${ }^{19}$

Die starke Konzentration auf das Thema Wirtschaft schien angesichts der dürftigen rotgrünen Bilanz ${ }^{20}$ und der angespannten wirtschaftlichen Lage naheliegend. Allerdings über-

16 So CDU-Wahlkampf-Experte Oliver Rösler, zitiert nach Thomas Breuer, Die Fernsehduelle der Spitzenkandidaten von SPD und CDU/CSU im Bundestagswahlkampf 2002, Dissertation, Bonn 2006, S. 238.

17 So wartete die Union etwa mit Slogans wie „Deutschland braucht den Wechsel”, „Besser für die Menschen” oder „Deutschlands Chancen nutzen” auf. Die SPD suchte mit ihren zwei Themenplakat-Serien hingegen die inhaltliche Abgrenzung. Auf dem einen Plakattyp reklamierte sie zunächst politikinhaltliche Positionen für sich (etwa: „Wir stehen für moderne Familienpolitik”) und schob dann jeweils die verunsichernde Frage nach: „Aber wofür stehen die Anderen?”, wobei mit diesen Anderen zumeist inhaltlich nur CDU und CSU gemeint sein konnten (teilweise aber auch die FDP). Bei dem zweiten Plakattyp wurde die (unterstellte) Position der „Anderen” explizit formuliert (zum Beispiel: „Für soziale Gerechtigkeit. Gegen sozialen Kahlschlag”). Auf beiden Plakattypen wurden die konstruierten Dichotomien durch Schwarz-auf-Weiß-Druck der SPDPosition und Weiß-auf-Schwarz-Druck der Gegenposition optisch scharf voneinander abgegrenzt; vgl. Christina Holtz-Bacha / Eva-Maria Lessinger, Politische Farbenlehre. Der Plakatwahlkampf 2005, in: Christina Holtz-Bacha (Hrsg.), a.a.O. (Fn. 1), S. 80 - 125, S. 98 f.

18 Vgl. Richard Hilmer / Rita Müller-Hilmer, Die Bundestagswahl vom 18. September 2005, Votum für Wechsel in Kontinuität, in: ZParl, 37. Jg. (2006), H. 1, S. 183 - 218, S. 213.

19 Von den fünf weiteren Kapiteln ist das umfangreichste ein unverbundenes Sammelsurium unter dem Titel „Wir gestalten ein lebenswertes Deutschland“ mit viereinhalb Seiten, das längste inhaltlich kohärente die Außenpolitik mit dreieinhalb Seiten. Bundesvorstand der CDU / Parteivorstand der CSU, Deutschlands Chancen nutzen. Wachstum. Arbeit. Sicherheit. Regierungsprogramm 2005-2009, Berlin 2005, http://www.cducsu.de/upload/regierungsprogramm.pdf (Abruf am 12. August 2009).

20 Vgl. Roland Sturm, Eine Bilanz mit Schatten? Die Arbeit der rot-grünen Regierung auf zentralen Politikfeldern, in: Eckhard Jesse / ders. (Hrsg.), Bilanz der Bundestagswahl 2005. Voraussetzungen, Ergebnisse, Folgen, Wiesbaden 2006, S. $71-82$. 
schätzte die Union die subjektive Relevanz der Arbeitslosigkeit für die Wahlentscheidung persönlich nicht tangierter Bürger ${ }^{21}$, zumal ihr hierbei nur jeder fünfte Wahlberechtigte eine Trendwende zutraute. Zugetraut wurde der CDU/CSU hingegen ein wirtschaftlicher Aufschwung, jedoch glaubten viele Befragte nicht, dass sie selbst von einem solchen profitieren würden. ${ }^{22}$ Übrig blieb in der Bevölkerung die Wahrnehmung eines unsozialen Kurses der Union in der Wirtschafts- und Steuerpolitik. ${ }^{23}$ Die SPD schürte erfolgreich Ängste vor sozialen Verwerfungen im Falle einer schwarz-gelben Regierungsübernahme. Die Figur des von Merkel in ihr Kompetenzteam berufenen ehemaligen Bundesverfassungsrichters Paul Kirchhof ermöglichte die Personifizierung dieser Befürchtungen. Kirchhofs persönliches Konzept einer Pauschalsteuer von 25 Prozent bei Streichung so gut wie sämtlicher Steuervergünstigungen zeitigte einen zunächst unterschätzten Effekt: die Aufweichung des programmatischen Profils der Union. ${ }^{24}$ Die zuvor abgeschlagene SPD zog im Laufe der Debatte in der Steuerkompetenz mit der Union gleich. ${ }^{25}$ Dennoch scheint es übertrieben, Kirchhofs Nominierung zur „Achillesferse des Unionswahlkampfes“ ${ }^{26}$ zu stilisieren, denn dies suggeriert, dass die Kampagne von CDU/CSU an keiner anderen Stelle „verwundbar“ gewesen wäre. Dies ist nicht einmal für das begrenzte Politikfeld Steuern der Fall, wie die Zustimmungsraten zur Kürzung der Pendlerpauschale und zur Senkung des Spitzensteuersatzes zeigen. ${ }^{27}$ Dass die Forcierung des Themas Steuern früher oder später Fragen der sozi-

21 Vgl. Dieter Roth / Andreas M. Wüst, Abwahl ohne Machtwechsel? Die Bundestagswahl 2005 im Lichte langfristiger Entwicklungen, in: Eckhard Jesse / Roland Sturm (Hrsg.), a.a.O. (Fn. 20), S. $43-70$, S. $56 \mathrm{f}$.

22 Vgl. Richard Hilmer / Rita Müller-Hilmer, a.a.O. (Fn. 18), S. 209.

23 Vgl. Matthias Jung / Andrea Wolf, a.a.O. (Fn. 13), S. 5.

24 Bei einer Umfrage gaben 71 Prozent der Befragten an, nicht zu wissen, woran man bei der Union in der Steuerpolitik genau sei, vgl. FAZ vom 20. September 2005, S. 7.

25 Vgl. Infratest dimap, ARD-DeutschlandTrend, September 2005 II, S. 6.

26 Hans Rattinger / Zoltan Juhasz, Die Bundestagswahl 2005. Neue Machtkonstellation trotz Stabilität der politischen Lager, München 2006, S. 7.

27 Vgl. Markus Klein, Jenseits von Distanz und Richtung. Die Verbundmessung von Politikpräferenzen im Vergleich mit dem Distanz- und dem Richtungsmodell des Wählens - empirische Befunde eines Methodenexperiments, in: PVS, 47. Jg. (2006), H. 4, S. 595 - 617, S. 610. Allerdings war die Idee gewagt, zu Beginn der heißen Wahlkampfphase einen Außenseiter als Finanzminister in spe zu präsentieren; vgl. Andreas Wüst, Ein verschossener Elfmeter und die Verpflichtung zum Erfolg, in: Einsichten und Perspektiven, Nr. 3/2005, S. 24 - 31, S. 26. Zu erklären ist diese Risikobereitschaft Merkels wohl dadurch, dass die CDU-Chefin durch Edmund Stoibers öffentlich zur Schau gestellte Unentschlossenheit, ob und welchen Posten er in einem Kabinett Merkel übernehmen würde, und die Kompetenz und Popularität von Friedrich Merz, dessen Rückzug Merkel sich zurechnen lassen musste, bei der Besetzung des Bereichs Wirtschaft im Kompetenzteam in die Defensive geraten war. Die Entscheidung für Kirchhof kann in dieser Situation als Befreiungsschlag begriffen werden (vgl. Stephan Eisel, a.a.O. (Fn. 4), S. 51) oder als machtpolitisches Kalkül Merkels, mit Hilfe Kirchhofs die Ambitionen Stoibers auf ein kombiniertes Wirtschafts- und Finanzministerium zu schwächen, den Finanzfachmann Merz auf Distanz zu halten und zugleich den Reformwillen der Union zu bekräftigen (vgl. Daniel Deckers, Vier Sieger, ein Verlierer?, in: FAZ vom 20. September 2005, S. 7). Ebenfalls bedenkenswert ist die Argumentation, dass Merkel nicht die nötige Autorität zur Legitimierung ihrer Reformmaßnahmen besessen habe und Kirchhof diese von außen liefern sollte (vgl. Johannes Leithäuser, Die Politik als ehrliches Handwerk, in: FAZ vom 14. September 2005, S. 3). Eine ähnliche Rolle wurde nolens volens auch Bundespräsident Horst Köhler zugewiesen (vgl. Albrecht von Lucke, Ruck ins Ressentiment, in: Blätter für deutsche und internationale Politik, 50 Jg. (2005), H. 9, S. 1031 - 1034, S. 1033). 
alen Gerechtigkeit auf die Tagesordnung bringen würde, war abzusehen. Die Vernachlässigung des sozialen Aspekts in einem als wirtschaftszentriert geplanten Wahlkampf geriet zum Hauptangriffspunkt gegen die Union.

\subsection{Lagerwahlkampf}

Nach dem Scheitern ihrer Eigenständigkeitsstrategie 2002 war die FDP bereits im Laufe der Wahlperiode an die Seite der Union zurückgekehrt. Union und FDP stellten ihre Einigkeit gegenüber dem getrennten Wahlkampf der Regierungsparteien ${ }^{28}$ symbolisch bedeutsam vor allem durch den „Wechselgipfel“ am 1. September 2005 heraus. Im Ergebnis profitierte vom gemeinsamen Lagerwahlkampf allerdings allein die FDP, und zwar auf Kosten der Unionsparteien: Gegenüber 2002 verloren diese im Saldo 1,1 Millionen Wähler an die Liberalen. Als Motive der Abwanderer werden in der Forschung die Verhinderung einer sich in den Umfragen abzeichnenden Großen Koalition und der Ausdruck einer Präferenz für Schwarz-Gelb ${ }^{29}$, die Forcierung des Reformkurses einer bürgerlichen Koalition und die Ablehnung der auch von der FDP für unnötig befundenen Erhöhung der Mehrwertsteuer diskutiert. ${ }^{30}$ Ungeachtet der konkreten Motive für die Wechselentscheidung wurde diese erleichtert durch „eine bisher ungekannte Distanzlosigkeit zwischen Union und FDP“31, die auch durch die Nominierung Kirchhofs begünstigt wurde. ${ }^{32}$ Der Eindruck, die CDU/ CSU sei nichts anderes als ein „gleichsam verlängerter Arm der FDP“33, verleitete nicht nur

28 Der Verzicht auf ein Bekenntnis zu Rot-Grün ermöglichte SPD und Grünen, sich stärker von Misserfolgen der Regierungszeit abzusetzen und in erster Linie das Programm der "gefühlten Regierung" zu thematisieren.

29 Gegen diese verbreitete These bringt Eckhard Jesse vor, mit der Wahl für die FDP habe der Wähler doch eine große Koalition nicht verhindern können, vielmehr wäre diese, hätte die FDP noch einen weiteren Prozentpunkt Stimmen aus den Reihen der Unionsanhänger erhalten, unter SPDFührung zustande gekommen. Die Angst, die FDP könne an der Fünfprozenthürde scheitern, sei „bar jeder Plausibilität“ gewesen, vgl. Eckhard Jesse, Der Ausgang der Bundestagswahl 2005 - ein halber Regierungswechsel, in: Politische Studien, 57. Jg. (2006), H. 1, S. 72 - 89. S. 77. So zutreffend diese Analyse ist, könnte dennoch dem - eventuell spontan entscheidenden - durchschnittlichen Wähler mit geringerem politstrategischem Reflexionsvermögen die Stimmabgabe für die FDP als probates Mittel zur Vermeidung einer großen Koalition eingeleuchtet haben.

30 Zur zwingenden Bedingung für eine Koalition wollten die Spitzenpolitiker der FDP die Mehrwertsteuerfrage aber nicht machen (vgl. FAZ vom 12. Juli 2005, S. 1; SZ vom 13. Juli 2005, S. 5). Mehr als eine Zweckbindung und/oder Verschiebung der Maßnahme hofften sie wohl gar nicht zu erreichen (vgl. FAZ vom 5. Juli 2005, S. 1 und vom 16. Juli 2005, S. 10; Spiegel Online vom 12. Juli 2005, http://www.spiegel.de/politik/deutschland/0,1518,364790,00.html (Abruf am 12. August 2009)). Die Position der FDP zur Mehrwertsteuerfrage wurde von den Wählern auch nur unzureichend perzipiert: Jeder fünfte Befragte wusste hierzu keine Angabe zu machen, von den übrigen vier Fünfteln nahm fast die Hälfte an, die FDP sei für eine Erhöhung, vgl. Markus Klein, a.a.O. (Fn. 27), S. 611.

31 Matthias Jung / Andrea Wolf, a.a.O. (Fn. 13), S. 6.

32 Westerwelle bezeichnete Kirchhof als „Verbündeten im Geiste“ (zitiert nach FAZ vom 19. August 2005, S. 11) und kokettierte, es sei ein Gerücht, dass dieser FDP-Mitglied sei - in der Tat hatten die Liberalen den Steuerfachmann zuerst „entdeckt“ und auf ihrem Parteitag im Mai 2005 reden lassen (vgl. Frankfurter Allgemeine Sonntagszeitung vom 28. August 2005, S. 3).

33 Berthold Löffler, Wahlkampf nur auf einem Bein. Hat die Union den Sieg verschenkt?, in: Die Politische Meinung, Nr. 10/2005, S. 29 - 33, S. 31. 
den reformbegeisterten Teil der Unionsanhänger, gleich das „Original zu wählen“, sondern stellte auch einen für eine Volkspartei mit einer heterogenen Wählerschaft verheerenden Imagetransfer dar. ${ }^{34}$ Insgesamt erwies sich der Lagerwahlkampf für die Union daher als fatal.

\subsection{Gesamtdeutscher Wahlkampf}

Die neuen Länder nahmen als zehnter von elf Unterpunkten des langen ersten Kapitels „Vorfahrt für Arbeit“ keine sehr prominente Position im Regierungsprogramm der Union ein. Auch inhaltlich wurden den Ostdeutschen speziell lediglich Allgemeinplätze angeboten. Im Gegensatz zu SPD, Linkspartei und Grünen hielt die Union die für eine Anhebung der Hartz IV-Sätze auf Westniveau erforderlichen 250 bis 300 Millionen Euro für nicht aufzubringen ${ }^{35}$, was ihr angesichts eines Haushaltvolumens von knapp 250 Milliarden Euro und der geplanten Absenkung des Spitzensteuersatzes von 42 auf 39 Prozent einmal mehr den Vorwurf sozialer Ungerechtigkeit einbrachte. Die Christdemokraten hatten verkannt, dass viele Ostdeutsche dem Thema Symbolkraft für eine Anerkennung ihrer Erfahrungen als gleichwertig zumaßen. Die von Merkel angebotene Anerkennung beschränkte sich auf Rhetorik. In ihren Wahlkampfreden lobte sie, wenn alle Deutschen so viel Veränderungsbereitschaft gezeigt hätten wie die Ostdeutschen, wäre man in einigen Fragen schon ein Stück weiter. ${ }^{36}$ Die Rolle der Avantgarde einer Politik der Kürzungen wollten die Ostdeutschen jedoch nicht verkörpern, wie die Demonstrationen gegen Hartz IV hinlänglich deutlich hätten machen sollen. Einige der von der Union geplanten Einschnitte, etwa bei den Arbeitnehmerrechten, der aktiven Arbeitsmarktpolitik und der Pendlerpauschale wiesen aber besondere Virulenz für Ostdeutschland auf. 70 Prozent der Ostdeutschen glaubten, dass ihnen ein Wahlerfolg Merkels schaden könne. ${ }^{37}$ Einen Heimbonus gab es daher für sie im Gegensatz zu früheren Kanzlerkandidaten nicht ${ }^{38}$, woran auch die von ostdeutschen CDUPolitikern geforderte ${ }^{39}$ stärkere Akzentuierung ihrer Herkunft wohl nichts geändert hätte. Die CDU schnitt 2005 in den neuen Bundesländern noch schlechter ab als 2002 mit dem dort unbeliebten Stoiber. Dazu trug dieser auch diesmal mit seiner Ostdeutschen-Schelte bei. ${ }^{40}$ Weder gegen ihn noch gegen ähnliche Äußerungen des baden-württembergischen

34 Vgl. Gerhard Hirscher, Ende der bürgerlichen Mehrheit? Die Oppositionsparteien CDU, CSU und FDP, in: Eckhard Jesse / Roland Sturm (Hrsg.), a.a.O. (Fn. 20), S. 83 - 118, S. 112 ff.; Matthias Jung / Andrea Wolf, a.a.O. (Fn. 13), S. 6.

$35 \mathrm{Vgl}$. FR vom 16. August 2005, S. 1.

36 Vgl. FAZ vom 17. August 2005, S. 3.

37 Vgl. Eveyln Finger, Der Ossi als Wessi, in: Die Zeit vom 25. August 2005, S. 35.

38 Vgl. Matthias Geis, Fremdeln in der Uckermark, in: Die Zeit vom 28. Juli 2005, S. 3.

39 Vgl. FAZ vom 21. Juli 2005, S. 1.

40 Stoiber erklärte auf einer Wahlkampfveranstaltung in Baden-Württemberg, er akzeptiere nicht, „dass der Osten bestimmt, wer in Deutschland Kanzler wird“, und setzte hinzu: „Die Frustrierten dürfen nicht über Deutschlands Zukunft bestimmen. "Bei einem Auftritt in Bayern sagte Stoiber, die CSU brauche ein gutes Ergebnis, um Defizite der CDU etwa in Sachsen und Sachsen-Anhalt auszugleichen: „Wir haben leider nicht überall so kluge Bevölkerungsteile wie in Bayern“ (zitiert nach SZ vom 12. August 2005, S. 1). 
Ministerpräsidenten Günther Oettinger ${ }^{41}$ schritt Merkel ein. Einzig den brandenburgischen Innenminister Jörg Schönbohm mahnte sie angesichts seiner These einer „Proletarisierung“ der ostdeutschen Gesellschaft ab. ${ }^{42}$ Die Befindlichkeiten der Wähler im Osten wurden der inneren Geschlossenheit der Union untergeordnet. Anstatt Stoiber für seinen Fauxpas zu rügen, antwortete Merkel den Bürgern mit dem Totschlagargument, der Unterschied zu vor 15 Jahren sei, dass heute jeder seine Meinung kundtun könne, ohne gleich hinter Gitter zu kommen. ${ }^{43}$

\section{Der Wahlkampf der kommunizierten Ehrlichkeit}

\subsection{Die Problematik der Ehrlichkeit im Wahlkampf}

Auf die offene Frage, was sie an den Parteien am meisten störe, nennt weit über die Hälfte der Befragten leere Versprechungen, Unehrlichkeit und Korruption. ${ }^{44}$ Zwei Drittel der Wähler ziehen einen Kandidaten, dem sie vertrauen, dessen Ziele ihnen aber nicht gefallen, einem Kontrahenten vor, dessen Ziele sie teilen, bei dem sie sich aber nicht sicher sind, ob er sich daran hält. ${ }^{45}$ Unbestritten ist, dass die Tragfähigkeit demokratischer Politik von der durch die Erkenntnis der Wirklichkeit geprägte Qualität der öffentlichen Diskussion abhängt. ${ }^{46}$ Je anspruchsvoller man die Kategorie der Ehrlichkeit definiert, desto mehr muss man allerdings zu der Auffassung gelangen, dass manche Unwahrheiten „ein zumindest normaler, wenn nicht gar ein legitimer Bestandteil politischen Handelns sind“ ${ }^{\text {“7 }}$. So gab schon Platon seinen Philosophenkönigen in der Politeia das Recht zu lügen, um den Idealstaat zu sichern. ${ }^{48}$ Machiavelli appellierte an seinen „Fürsten“ explizit, sein Wort nur dann zu halten, wenn ihm daraus keine Nachteile erwüchsen. ${ }^{49}$ Selbst Hannah Arendt schrieb, niemand habe je die Wahrhaftigkeit zu den politischen Tugenden gerechnet. ${ }^{50}$ Aufgrund ihres größeren politischen Verständnisses neigen Politiker zu paternalistischen Lügen, also

41 Oettinger hatte Anfang August in Pforzheim geäußert: „Die Linken und die Mutlosen im Osten Deutschlands dürfen nicht entscheiden, wie Deutschland regiert wird“, zitiert nach FAZ vom 13. August 2005, S. 1.

$42 \mathrm{Vgl}$. FR vom 5. August 2005, S. 1.

43 Vgl. Eveyln Finger, a.a.O. (Fn. 37), S. 35.

44 Vgl. Hans Rattinger, Die Parteien. Ungeliebt - aber ohne Alternative, in: Jürgen W. Falter / Oscar W. Gabriel / ders. / Harald Schoen (Hrsg.), Sind wir ein Volk? Ost- und Westdeutschland im Vergleich, München 2006, S. 82 - 106, S. 96.

45 Vgl. Hans Mathias Kepplinger / Marcus Maurer, Abschied vom rationalen Wähler. Warum Wahlen im Fernsehen entschieden werden, Freiburg / München 2005, S. 171.

$46 \mathrm{Vgl}$. Hannah Arendt, Wahrheit und Lüge in der Politik. Zwei Essays, München 1972, S. 46; Julian Nida-Rümelin, Demokratie und Wahrheit, München 2006, S. 7; Georg-Berndt Oschatz, Müssen Politiker lügen? Demokratie zwischen Diskurs und Irreführung, in: Otto Depenhener (Hrsg.), Recht und Lüge, Münster 2005, S. 45 - 56, S. 51 ff.; Jean-François Revel, Die Herrschaft der Lüge. Wie Medien und Politiker die Öffentlichkeit manipulieren, Wien / Darmstadt 1990, S. $14 \mathrm{f}$.

47 Claus Offe, Die Ehrlichkeit politischer Kommunikation. Kognitive Hygiene und strategischer Umgang mit der Wahrheit, in: Vorgänge, 43. Jg. (2004), H. 3, S. 28 - 38, S. 32.

48 Vgl. Platon, Der Staat, Stuttgart 1999 [zuerst um 370 v. Chr.], S. 165.

49 Vgl. Niccolò Machiavelli, Der Fürst, Frankfurt 2001 [zuerst 1532], S. 70.

50 Vgl. Hannah Arendt, a.a.O. (Fn. 46), S. 44. 
zu Täuschungen der Bürger „zu ihrem eigenen Besten“51. Auf lange Sicht ist es nicht zwingend schädlich für Regierungen, nach der Wahl etwas anderes zu tun als zuvor angekündigt; sie können dabei auf die Vergesslichkeit der Wähler und/oder den Erfolg der veränderten Politik setzen. ${ }^{52}$ Das Dilemma solider Politik liegt umgekehrt darin, dass sie erst langfristig vertrauensbildend wirkt, ihr diese Zeitspanne aber gar nicht zur Verfügung steht, wenn sie mangels hinreichender Vermittlung ihrer Politik kurzfristig abgewählt wird. ${ }^{53}$ Zeiten ökonomischer Knappheit stellen die Politik vor die zusätzliche Schwierigkeit, Mehrheiten für unpopuläre Entscheidungen zu organisieren, da sich die zunehmenden Verteilungskonflikte nicht mehr durch Zuwächse konsensual schlichten lassen. ${ }^{54}$

Wenn auch zwei Drittel der Bürger wahrhaftige Politiker wollen, so sträubt sich doch eine gleich große Mehrheit gegen Veränderungen zum eigenen Nachteil. ${ }^{55}$ Politik bleibt „Streit um Macht, nicht ein moralischer Schönheitswettbewerb“, es geht daher „nicht um die Wahrheit, sondern um die Mehrheit" ${ }^{\text {" }}$. Claus Offe rügt die „populistische Lüge der Authentizität“: Die Leugnung des „genuin strategischen und insofern ,unehrlichen' Charakters politischen Handelns" sei selbst unehrlich. ${ }^{57}$ Peter Kemper argumentiert, das Kriterium der Ehrlichkeit liefere den politischen Prozess der Gefahr der Personalisierung zu Gunsten einiger „Autoritäten der Glaubwürdigkeit“ aus und reduziere so vorschnell die Komplexität der Zusammenhänge - worin vielleicht auch seine eigentliche Faszination liege. ${ }^{58}$ Die Hegemonie über die Interpretation von „Wirklichkeit“ zeitigt laut Franz Walter einen Vorsprung im politischen Wettbewerb. ${ }^{59}$ Der Kampf um diese Deutungshoheit bringt mit zunehmender Entideologisierung Lügen hervor, weil Erfolg davon abhängt, was man die Anderen glauben machen kann. ${ }^{60}$ Allerdings halten einige Autoren Ehrlichkeit für kaum kommunizierbar, ihr Hervorheben nähere gerade den Zweifel an ihr und werfe zudem die Frage auf, ob dies in Ermangelung sonstiger positiver Eigenschaften geschehe. ${ }^{61}$ Da die Bürger den Typ des ehrlichen Politikers als „zu gut“ für das nun mal „schmutzige Geschäft" Politik abqualifizierten und erschreckten, wenn dieser von ihnen seinerseits Ehrlichkeit einfordere, machten sie sich mitverantwortlich für die Unehrlichkeit in der Poli-

51 Vgl. Sissela Bok, Lügen. Vom täglichen Zwang zur Unaufrichtigkeit, Reinbek 1980, S. 202 f.

52 Vgl. Frank Decker, Höhere Volatilität bei Landtagswahlen. Die Bedeutung bundespolitischer ,Zwischenwahlen', in: Eckhard Jesse / Roland Sturm (Hrsg.), a.a.O. (Fn. 20), S. 259 - 279, S. 262.

53 Vgl. Hans-Martin Schönherr-Mann, Wieviel Vertrauen verdienen Politiker?, in: APuZ, B 15 / 16 (2002), S. 3 - 5, S. 5.

54 Vgl. Karl-Rudolf Korte, Was entschied die Bundestagswahl 2005?, in: APuZ, B 51 / 52 (2005), S. $12-18$, S. 16.

55 Vgl. Georg-Berndt Oschatz, a.a.O. (Fn. 46), S. 52.

56 Theo Sommer, Jetzt mal ehrlich, in: Die Zeit vom 18. August 2005, S. 1.

57 Vgl. Claus Offe, a.a.O. (Fn. 47), S. 33 f.

58 Vgl. Peter Kemper, Vorwort, in: ders. (Hrsg.), Opfer der Macht? Müssen Politiker ehrlich sein?, Frankfurt am Main / Leipzig 1993, S. 7 - 12, S. 10.

59 Vgl. Franz Walter, Die ungleichzeitige Wirklichkeit. Eine Besichtigung der deutschen Gesellschaft im Jahr 2005, in: Internationale Politik, 60. Jg. (2005), H. 10, S. 6 - 13, S. 9.

60 Vgl. Claus Offe, a.a.O. (Fn. 47), S. 29.

61 Vgl. Norbert Bolz, Politik als ob oder Die Lizenz zu lügen, in: Peter Kemper (Hrsg.), a.a.O. (Fn. 58), S. 58 - 70, S. 65; Niklas Luhmann, Die Ehrlichkeit der Politiker und die höhere Amoralität der Politik, in: Peter Kemper (Hrsg.), a.a.O. (Fn. 58), S. 27 - 41, S. 30; Thomas H. Macho, Container der Aufmerksamkeit, in: Peter Kemper (Hrsg.), a.a.O. (Fn. 58), S. 194 - 207, S. 195; Irmtaud Tarr Krüger, Von der Unmöglichkeit, ohne Lügen zu leben, Zürich 1997, S. 50. 
tik. ${ }^{62}$ Zum Lügen gehören immer zwei: „einer, der lügt, und einer, der sich entweder aus Naivität und Mangel an demokratischem Argwohn belügen lässt oder aus Zynismus und Indifferenz selbst erwiesene Lügen für Bagatellangelegenheiten hält"63.

\subsection{Anforderungen an einen Wahlkampf der kommunizierten Ehrlichkeit}

Bei der Entscheidung für eine Wahlkampfstrategie der kommunizierten Ehrlichkeit sind neben den bereits erörterten Aspekten, insbesondere der Schwierigkeit der Selbstinszenierung als „ehrliche Haut“, folgende Aspekte zu beachten:

Erstens ist Moral durch Kompromisslosigkeit gekennzeichnet, die eine situative Abwägung ausschließt: „Moralität ist nämlich in der Reihe der zu berücksichtigenden Aspekte einer Güterabwägung nicht ein besonders schönes oder wertvolles dieser Güter, sondern die Moralität ergibt sich (oder nicht) aus der Reihenfolge aller übrigen Aspekte. "64 Ehrlichkeit als moralische Kategorie trägt also einen Absolutheitsanspruch in sich: Wirklich ehrlich zu sein bedeutet hundertprozentige Ehrlichkeit. Eine lediglich relative Ehrlichkeit ist streng genommen schon unehrlich.

Umso fataler kann es zweitens sein, wenn man immer wieder seine Ehrlichkeit postuliert und dann doch nicht an die Höhe der Erwartungen heranreicht. Wer explizit seine eigene Ehrlichkeit herausstellt, riskiert, von der Öffentlichkeit diesbezüglich an einem strikteren Maßstab gemessen zu werden als politische Mitbewerber, die sich nicht auf diese Weise zu profilieren versuchen. Marcel Proust bemerkte, dass das Generieren einer solchen erhöhten Erwartungshaltung gerade zum Gegenteil der versprochenen Tugendhaftigkeit führe: „Proklamiert zu haben (als Führer einer politischen Partei oder was sonst immer), daß es abscheulich ist zu lügen, zwingt in der Mehrzahl der Fälle dazu, mehr als die anderen zu lügen, ohne daß man deswegen die feierliche Maske oder die erhabene Tiara der Gesinnungstreue ablegen darf." 65

Drittens bietet man nämlich durch Offenheit in Bezug auf geplante unpopuläre Maßnahmen eine willkommene Angriffsfläche für politische Gegner. Diese können nicht ihrerseits auf eine ehrliche Wahlkampfführung verpflichtet werden. Die Frage lautet: „Wie kann man sich selbst unerschütterlich an die Moral halten, wenn andere es nicht tun?"66 Strikte Wahrhaftigkeit kann man sich nur leisten, wenn man sicher ist, dass die Mitbewerber dieser Norm ebenfalls folgen beziehungsweise dass deren Unehrlichkeit von den Wählern bei Aufdeckung scharf sanktioniert wird. ${ }^{67}$ Auf die Unehrlichkeit des Gegners kann man nämlich nur sehr beschränkt reagieren. Ist die Losung von der Ehrlichkeit erst einmal ausgegeben, kann man die Strategie im Prinzip nicht mehr offiziell wechseln, anders als zum Beispiel im Falle des Versprechens, auf Negative Campaigning zu verzichten: Wird man nach Abgabe

62 Vgl. Klaus Staeck, Die Opfer als Täter, in: Peter Kemper (Hrsg.), a.a.O. (Fn. 58), S. 97 - 111, S. $102 \mathrm{ff}$.

63 Vgl. Claus Offe, a.a.O. (Fn. 47), S. 38.

64 Reinhard Löw, Moral und Moralisierung, in: Peter Kemper (Hrsg.), a.a.O. (Fn. 58), S. 139 - 151, S. 141 (Hervorhebung im Original).

65 Marcel Proust, Auf der Suche nach der verlorenen Zeit, Bd. V: Die Gefangene (1923), Frankfurt am Main / Zürich 1956, S. 265.

66 Niklas Luhmann, a.a.O. (Fn. 61), S. 28 (Hervorhebung im Original).

67 Vgl. Claus Offe, a.a.O. (Fn. 47), S. 33. 
eines solchen Versprechens vom Gegner in unerträglicher Weise mit Schmutzkampagnen belegt, kann immer noch ein Schlussstrich gezogen und mit Bedauern die Abkehr von der ursprünglichen lauteren Absicht verkündet werden, wofür dem Gegner gegenüber dem (Wahl-)Publikum die Schuld angelastet werden kann. Eine Strategie der Ehrlichkeit kann hingegen lediglich intern aufgekündigt werden, was einen indes nicht davor bewahrt, weiterhin extern an der offiziell fortbestehenden Aussage gemessen zu werden.

\subsection{Die Entscheidung der Union für die Ehrlichkeitsstrategie}

Der Ehrlichkeitsstrategie im Bundestagswahlkampf 2005 lag eine bewusste Entscheidung zugrunde. Weder wurde sie mehr oder weniger unüberlegt aus Vorwahlkämpfen übernommen, noch kann sie als unreflektierter Niederschlag im Wahlprogramm von persönlicher Ehrlichkeitsliebe des führenden Unionspersonals bei der Wähleransprache aufgefasst werden ${ }^{68}$; darauf verweist auch das Faktum ihrer aktiven Kommunikation. Überzeugungstäterschaft in puncto Ehrlichkeit ist eher den Grünen im Bundestagswahlkampf 1998 zuzurechnen, die sich mit den offenen Forderungen nach Ökosteuer, Benzinpreiserhöhung und Tempolimit bei vielen Wählern unbeliebt machten, ohne sich für die Offenheit dieser Ankündigung zu preisen.

Der Union stand 2005 eine andere Wahlkampagne Pate: jene, mit der Jürgen Rüttgers im Mai in Nordrhein-Westfalen erfolgreich gewesen war. ${ }^{69}$ Stoiber deutete Rüttgers' Sieg als Beweis, dass die Union mit Ehrlichkeit Wahlen gewinnen könne ${ }^{70}$, unabhängige Beobachter bemängelten hingegen die Konturlosigkeit dessen Kampagne. ${ }^{71}$ Die unpopulären Maßnahmen in Rüttgers' Programm beschränkten sich auf die Halbierung der Steinkohlesubventionen und einen Personalabbau im öffentlichen Dienst, insbesondere in der engeren Landesverwaltung. Mit der ersten Forderung schnitt sich die Union kaum ins eigene Wähler-Fleisch, und die zweite genoss in der breiten Bevölkerung durchaus Popularität. Rüttgers' Wahlkampf bot also kaum Angriffsfläche, worin auch seine Attraktivität für Merkel lag. ${ }^{72}$ Nach Darstellung des „Spiegel“ verabredete Merkel mit Volker Kauder, es ebenfalls bei einigen wenigen klaren Ankündigungen zu belassen, die dann gleichsam als „Leuchttürme“ die Vagheit des restlichen Programms „überstrahlen“ sollten. Der Wähler solle wie bei einem Lackmustest von einzelnen Ausweisen der Ehrlichkeit auf das Gesamtkonzept schließen. ${ }^{73}$ Von dieser Strategie versprach man sich zunächst Vorteile am Tag der Wahl, wenn die Wähler die Ehrlichkeit oder zumindest deren Anschein honorieren würden ${ }^{74}$, sodann aber auch

68 So erklärte Norbert Röttgen ausdrücklich, es handele sich bei Ehrlichkeit in der Politik nicht um eine naive Forderung, sondern um eine Erfolgsstrategie, vgl. Spiegel Online vom 2. Juli 2005, http://www.spiegel.de/politik/deutschland/0,1518,363283,00.html (Abruf am 12. August 2009).

69 Vgl. Der Spiegel vom 30. Mai 2005, S. 37.

70 Vgl. Die Welt vom 21. Juni 2005, S. 2.

71 Vgl. Karl-Rudolf Korte, Die Botschaft aus Düsseldorf, in: Internationale Politik, 60. Jg. (2005), H. 7, S. 58 - 59, S. 59; Der Spiegel vom 30. Mai 2005, S. 37; Der Spiegel vom 20. Juni 2005, S. 27; Der Spiegel vom 1. Oktober 2005, S. 29; Welt am Sonntag vom 7. August 2005, S. 4.

72 Vgl. Der Spiegel vom 6. Juni 2005, S. 42.

73 Vgl. Der Spiegel vom 20. Juni 2005, S. 27.

74 Vgl. Bernd Ulrich, Die Radikalen kommen, in: Die Zeit vom 25. August 2005, S. 1. 
für die vier darauf folgenden Jahre, gewissermaßen als Präventivmaßnahme gegen postelektorale Frustration. ${ }^{75}$ Eine solche Vorbeugungsstrategie kann als Lehre aus der Unehrlichkeit der Regierungskoalition im Bundestagswahlkampf 2002 interpretiert werden, unter der diese die gesamte Wahlperiode lang gelitten hatte. ${ }^{76}$ Wohl nicht zufällig waren mit Roland Koch, Edmund Stoiber und Ole von Beust drei Ministerpräsidenten starke Befürworter eines Ehrlichkeitskurses; ihnen standen Anfang 2008 beziehungsweise im Herbst 2008 Landtagswahlen bevor, die für die Wähler Sanktionsmöglichkeiten gegenüber einer offenkundiger Wahllügen überführten Unionsregierung dargestellt hätten. ${ }^{77}$ Ein „Blut-Schweiß-undTränen“-Kurs bei der für Schwarz-Gelb ohnehin scheinbar kaum noch verlierbaren Bundestagswahl schien in dieser Hinsicht zumindest für diese Akteure, wenn nicht für die gesamte Partei, selbst dann als eine gute Investition in die Zukunft, wenn er einige Stimmen kosten sollte. Mit Steuersenkungsversprechen glaubte die Unionsspitze angesichts der Debatte über leere öffentliche Kassen bei den Wählern immer weniger Glauben und Anklang zu finden. Statt „Steuersenkung“ sollte „Ehrlichkeit“ zum neuen Zauberwort im Wahlkampf werden. ${ }^{78}$

Rot-Grün diente aber nicht nur als warnendes Beispiel im Umgang mit der Wahrheit, sondern zugleich als Kontrastfolie. „Entweder [ein] Weiter so mit Rot-Grün, mit Täuschungen und gebrochenen Versprechungen "79 oder ein Neuanfang mit der ehrlichen Union: Die ausschließliche Charakterisierung der politischen Alternative mittels rein moralischer Kategorien in dieser Interview-Äußerung Merkels dient im Sinne Kempers der radikalen Reduktion der Komplexität und der Selbststilisierung der Union zur einzigen Autorität der Glaubwürdigkeit und ihrer Kanzlerkandidatin zur „ehrlichsten Politikerin Deutschlands“, als die sie sich bei ihren Wahlkampfauftritten ankündigen ließ. Diese wie erläutert nicht unkomplizierte Selbstinszenierung als „ehrliche Haut“ stützte Merkel durch die vertrauensbildenden Mittel der Dämpfung zu kühner Hoffnungen sowie der dosierten Selbstkritik. ${ }^{80}$ Merkels stetige Verwendung von Sätzen wie „Ich muss Ihnen da die Wahrheit sagen, und die heißt ..." bedeutet im Sinne Franz Walters das Anstreben der elektoral wirkungsmächtigen Hegemonie über die Interpretation der Wirklichkeit.

Resümierend lässt sich festhalten: Die Union glaubte, sich angesichts des Umfragevorsprungs die Ehrlichkeitsstrategie leisten zu können, aus der sich für sie deutlich mehr Chancen als Risiken zu ergeben schienen. Bestenfalls durfte sie sich hiervon elektorale Zuwächse als Autorität der Glaubwürdigkeit und Inhaberin der Deutungshoheit über die Wirklichkeit sowie eine durch die weitgehende Vorablegitimation ihres künftigen Regierungshan-

75 Vgl. Christian Reiermann, Vorsorge gegen den Wahlkater, in: Welt am Sonntag vom 5. Juni 2005, S. 6.

76 Vgl. Matthias Jung / Andrea Wolf, a.a.O. (Fn. 13), S. 5.

77 Vgl. Der Spiegel vom 6. Juni 2005, S. 42. Stoiber äußerte dies auch explizit, vgl. Der Spiegel vom 13. Juni 2005, S. 45.

78 Vgl. Thomas Kröter, Ehrlichkeit heißt das neue Zauberwort, in: FR vom 2. Juni 2005, S. 3.

79 So Angela Merkel, zitiert nach SZ vom 20. Juli 2005, S. 7.

80 Vgl. Johannes Leithäuser, Die Politik als ehrliches Handwerk, in: FAZ vom 14. September 2005, S. 3. Als Beispiel für die Dämpfung zu kühner Hoffnungen führt Leithäuser die Äußerung Merkels an, auch die Union könne nicht von einem Tag auf den anderen alles verändern. Die dosierte Selbstkritik bestand im Eingeständnis, auch unter Kohl seien Wahlversprechen nicht gehalten worden. Weniger wohlwollend spricht Evelyn Finger von „Selbsterhöhung durch Selbstbezichtigung", vgl. Evelyn Finger, a.a.O. (Fn. 37). 
delns innenpolitisch ruhige Wahlperiode erhoffen. Schlechtestenfalls schien sie lediglich für die Regierungsbildung unerhebliche Stimmenverluste in Rechnung stellen zu müssen, die zudem noch durch das Ausbleiben einer Abstrafung bei den kommenden Landtagswahlen und der nächsten Bundestagswahl überkompensiert würden.

\section{3. (Un-)Ehrlichkeit im Regierungsprogramm}

Nach ihrer Nominierung zur Kanzlerkandidatin kündigte Merkel ein Programm „mit dem Mut zur Ehrlichkeit“ an: Es würden „kein Problem schöngeredet“, „keine Allwissenheit vorgetäuscht“ und keine „Patentrezepte formuliert, wo es keine Patentrezepte gibt“ 81 .

\subsection{Die Mehrwertsteuer als plakative Zumutung}

Der alles überstrahlende Leuchtturm der Ehrlichkeit, das Lackmuspapier im Unionsprogramm, die „plakative Zumutung " 82 war die Ankündigung der Erhöhung der Mehrwertsteuer von 16 auf 18 Prozent. Merkel betonte in ihren Wahlkampfreden, in denen dies teilweise ihre einzige konkrete Ankündigung darstellte, sie wolle dies als Ausweis ihrer Ehrlichkeit verstanden wissen. ${ }^{83}$ Wie unspektakulär dies eigentlich war, zeigte schon der geringe Anklang der gegen die „Merkelsteuer“ gerichteten SPD-Kampagne. ${ }^{84}$ Zumindest wurde die Bekenntnisbereitschaft der Union dadurch erleichtert, dass zwei Drittel der Wähler von einer SPD-geführten Bundesregierung ebenfalls eine Mehrwertsteuererhöhung erwarteten und nur ein Viertel den anderslautenden Erklärungen der SPD traute. ${ }^{85}$ Schon vor der Neuankündigung hatten sich in beiden Volksparteien Verfechter einer Erhöhung vorgewagt, waren aber von den Parteispitzen zurechtgewiesen worden. ${ }^{86}$ Noch wenige Tage vor Vorstellung des Regierungsprogramms hatte Merkel erklärt, die Senkung der Arbeitslosenversicherungsbeiträge sei auch ohne Mehrwertsteueranhebung finanzierbar, und sogar erwogen, eine Garantie für das Einfrieren des Mehrwertsteuersatzes abzugeben. ${ }^{87}$ Bei einem Test des Themas in Fokusgruppen wurde nämlich die Erhöhung der Mehrwertsteuer sofort geglaubt, die Arbeitsplätze schaffende Wirkung einer Absenkung der Lohnnebenkosten jedoch nicht ohne weiteres. Allerdings hatte dieser Test auch ergeben, dass die Wähler der Ankündigung eines Verzichts auf eine Mehrwertsteuererhöhung zutiefst misstraut hätten. ${ }^{88}$ Auch die Umfragen zeigten schon zu diesem Zeitpunkt, dass 82 Prozent der Wähler bei einem CDU/CSU-Wahlsieg mit einer Mehrwertsteuererhöhung rechneten. ${ }^{89}$ In dieser Gemengelage bot es sich für die Union an, diese auch mit ostentativer Offenheit anzukündigen, damit die Wähler mit einer unionsgeführten Regierung nicht nur die unbeliebte Maß-

81 Zitiert nach FAZ vom 31. Mai 2005, S. 1.

82 Vgl. Bernd Ulrich, Die Radikalen kommen, a.a.O. (Fn. 74).

83 Vgl. FAZ vom 15. August, S. 33 und vom 17. August, S. 3.

84 Vgl. Jens Schneider, Grenzen der Ehrlichkeit, in: SZ vom 2. September 2005, S. 4.

85 Vgl. Forschungsgruppe Wahlen, Politbarometer Juni 2005 I.

86 Vgl. SZ vom 13. Mai 2005, S. 4.

87 Vgl. FAZ vom 5. Juli 2005; Der Spiegel vom 11. Juli 2005, S. 41.

88 Vgl. Der Spiegel vom 18. Juli 2005, S. 23 f.

89 Vgl. Forschungsgruppe Wahlen, a.a.O. (Fn. 85). 
nahme verbinden würden, sondern auch deren ehrliche Ankündigung im Wahlkampf. Aufgrund der bereits erfolgten Diskussion war im Falle der Mehrwertsteuererhöhung die resignative Aufnahmebereitschaft der Wähler stark ausgeprägt, das elektorale Terrain also weiter geebnet als für jede andere in Frage kommende plakative Zumutung. Zudem hoffte die Union, durch die Verknüpfung des ohnehin erwarteten Übels Mehrwertsteuererhöhung mit der Absenkung der Arbeitslosenversicherungsbeiträge, die zugleich eine finanzielle Kompensation der sozialversicherungspflichtig beschäftigten Bevölkerungsteile bedeutete, die politischen Kosten ihrer Ehrlichkeitskampagne überschaubar halten zu können. Durch die interne Auseinandersetzung über den Verteilungsschlüssel der Mehrwertsteuererhöhung geriet die Absenkung der Beiträge zur Arbeitslosenversicherung allerdings in den Hintergrund. ${ }^{90}$ Der übliche Verteilungsschlüssel der Umsatzsteuer als Gemeinschaftssteuer sieht eine hälftige Aufteilung zwischen Bund und Ländern vor. ${ }^{91}$ Von den etwa 16 Milliarden Euro zusätzlicher Einnahmen durch eine Anhebung des Steuersatzes um zwei Prozentpunkte hätten dem Bund insofern gerade einmal acht Milliarden zugestanden. Mit ihrer Forderung, die gesamten Mehreinnahmen für Sozialreformen zu verwenden, konnte sich Merkel gegenüber den Ministerpräsidenten der Union nicht durchsetzen, und das Problem wurde auf die Zeit nach der Wahl verschoben. Die Nichtfestlegung des Länderanteils ${ }^{92}$ hebelte angesichts der Kosten der anvisierten Beitragssenkung von circa 15 Milliarden Euro die vermeintlich ausgeglichene Bilanz des Programms aus. Die geplanten Einsparungen bei den Arbeitsfördermaßnahmen blieben ebenfalls vage.

\section{2. (Un-)Ehrlichkeit bei weiteren programmatischen Vorhaben}

Ein vielleicht weniger starkes Glaubwürdigkeitssymbol als die Mehrwertsteuererhöhung stellte die geplante Vereinfachung der Einkommensteuer dar. Die Absenkung der Sätze sollte mit Streichungen oder Einschränkungen von Steuerbefreiungen, -vergünstigungen und Ausnahmetatbeständen „in gleichem Umfang“ einhergehen. Ausdrücklich genannt wurden das Schließen von Steuerschlupflöchern, die Reduzierung der Pendlerpauschale und der Abbau der Steuerfreiheit von Zuschlägen für Sonn-, Feiertags- und Nachtarbeit. Allerdings hätten sich die Kosten der niedrigeren Steuersätze und höheren Freibeträge auf 14 Milliarden Euro belaufen, die Gegenfinanzierungsmaßnahmen aber lediglich acht Milliarden eingebracht. ${ }^{93}$ Dennoch war die Ankündigung dieser Maßnahmen in gewisser Weise mutiger - weil weniger vorhersehbar - als jene der Mehrwertsteuererhöhung. Im Hinblick auf das auch in sich geschlossenere Gesamtpaket Einkommensteuersenkung und -vereinfachung

90 Vgl. Michael H. Spreng, Wahlkampf als eiskalte Polarexpedition, in: Forschungsjournal Neue Soziale Bewegungen, 19. Jg. (2006), H. 1, S. 5 - 11, S. 9.

91 Vgl. Art. 106 Abs. 3 GG. Der 2,2-prozentige Anteil der Gemeinden kann hier vernachlässigt werden.

92 In der Presse wurde über einen Länderanteil von 25 Prozent spekuliert; vgl. SZ vom 11. Juli 2005, S. 3.

93 Vgl. Der Spiegel vom 1. August 2005, S. 31. Davon hätten die 1,6 bis 1,9 Milliarden Euro aus der Steuerfreiheit der Zuschläge für Sonn-, Feiertags- und Nachtarbeit erst 2012 voll ausgeschöpft werden können. Aus dem anvisierten gestaffelten Abbau der Vergünstigung hätten zum Zeitpunkt der Einführung der Steuerreform Anfang 2007 gerade einige hunderttausend Euro zur Verfügung gestanden. 
relativiert sich dieser Mut allerdings, denn die Union glaubte vermitteln zu können, dass jeder Einzelne unter dem Strich profitieren würde. In Bezug auf den Kindergrundfreibetrag von 8.000 Euro propagierte das Wahlprogramm ein Plus von 5.000 Euro für eine Arbeitnehmerfamilie (mit zwei Kindern bis zu einem Jahreseinkommen von 38.200 Euro), realistisch erschienen lediglich 600 bis 700 Euro. ${ }^{94}$

Ehrlicher war die Union mit ihrer Ankündigung, den Kündigungsschutz zu lockern. Moniert werden könnte höchstens die - in der Forschung durchaus umstrittene ${ }^{95}-$ Behauptung, dies führe automatisch zu mehr Beschäftigung. Streng genommen handelte es sich um das Präsentieren eines Patentrezepts, wo es keines gibt. Bei der solidarischen Gesundheitsprämie wurden weder Einführungszeitpunkt noch Höhe, Kosten und Gegenfinanzierung spezifiziert. Die einzige Konkretisierung blieb, dass niemand bei der Einführung der Prämie mehr als bisher zahlen müsse, da für Versicherte mit niedrigem Einkommen ein sozialer Ausgleich vorgesehen sei. In einem Interview avisierte Merkel die Prämie für 2007 oder 2008, zu ihrer Finanzierung würden 13 Milliarden Euro benötigt, davon würden sieben durch die Senkung des Spitzensteuersatzes lediglich auf 39 statt wie ursprünglich geplant 36 Prozent aufgebracht. Der Hinweis darauf, wofür diese Mittel zu einem früheren Zeitpunkt - ohne tragfähige Gegenfinanzierung - ausgegeben werden sollten, ersetzte die Benennung von Quellen, aus denen sie nach der Wahl hätten entnommen werden können. Die restlichen sechs Milliarden sollten sich durch Einsparungen bei der Arbeitsmarktförderung ergeben ${ }^{96}$ - diese sollten allerdings auch schon für die Senkung des Beitrages zur Arbeitslosenversicherung herhalten. Zudem bezifferten andere Schätzungen die Kosten auf bis zu 23 Milliarden. ${ }^{97}$ Die Ersetzung des ursprünglichen, bedrohlich wirkenden Begriffs der „Kopfpauschale“ durch das klobige Wortkonstrukt „solidarische Gesundheitsprämie" mutete wie ein Etikettenschwindel an, ohne den Ursprungsbegriff aus der Erinnerung der Wähler tilgen zu können. Der Kombilohn wurde in fünf Zeilen abgehandelt, Ausgestaltung und Finanzierung blieben auch hier völlig unklar.

Die Verlängerung der Laufzeiten der Kernkraftwerke sollte den Wählern schmackhaft gemacht werden, indem ihnen in der Folge niedrigere Strompreise in Aussicht gestellt wurden. Durch den geringen Anteil der Erzeugungskosten an den Gesamtkosten sowie der Atomkraft an der gesamten Stromerzeugung wäre eine Preissenkung entsprechend der höheren Rendite der Konzerne für den Verbraucher aber kaum spürbar gewesen. ${ }^{98}$

Die Union kritisierte, der rot-grünen Regierung sei keine nachhaltige Sanierung der Rentenversicherung gelungen, in der darum bereits Ende 2007 eine neue Milliardenlücke drohe. Selbst schloss sie aber sowohl Rentenkürzungen als auch Beitragsanhebungen aus, im Gegenteil sollte sogar ein Kinderbonus in Form einer Beitragsermäßigung um 50 Euro monatlich eingeführt werden, finanziert durch die Abschaffung der Eigenheimzulage. Die Lebensarbeitszeit sollte nur durch einen früheren Berufseintritt infolge kürzerer Ausbildungszeiten verlängert werden, eine schrittweise Anhebung des Renteneintrittsalters kom-

94 Für eine nähere Erläuterung vgl. Focus vom 18. Juli 2005, S. 26 f.

95 Vgl. zum Beispiel Wolfgang Däubler, Die Mär vom rigiden Kündigungsschutz, in: Mitbestimmung, Nr. 3/2003, S. 37 - 39, S. 39; Renate Neubäumer, Der gesetzliche Kündigungsschutz aus ökonomischer Sicht, in: Sozialer Fortschritt, 55. Jg. (2006), H. 2/3, S. 25 - 34, S. 33.

96 Vgl. Die Welt vom 13. Juli 2005, S. 3.

97 Vgl. Stern vom 13. Juli 2005, S. 59.

98 Vgl. Focus vom 18. Juli 2005, S. 30. 
me erst in Frage, sobald es die Bedingungen auf dem Arbeitsmarkt erlaubten. In puncto Ehrlichkeit ist hier sogar „eine enttäuschende Rolle rückwärts“99 festzustellen, denn die Union hatte die Rente mit 67 schon beschlossen.

Im Bundeshaushalt machten CDU/CSU ein strukturelles Defizit zwischen 50 und 60 Milliarden Euro aus. Für 2013 wurde eine Reduzierung der Neuverschuldung auf null mittels einer „ehrlichen, nachhaltigen Haushaltspolitik“ angekündigt. Kein Politikfeld könne ex ante von Einsparungen ausgeschlossen werden, konkret genannt wurde aber nur der Auslauf des subventionierten Bergbaus. Über ein Konsolidierungsprogramm sollte erst nach dem Regierungswechsel ein Konvent aus Bund, Ländern und Kommunen beraten. Merkel machte „erhebliche Einsparmöglichkeiten“ bei Subventionen und Hartz IV aus, ohne dies weiter auszuführen. ${ }^{100}$ Zudem wurde der Subventionsabbau gleich mehrfach verplant: zur Sanierung der Staatsfinanzen, zur Senkung der Steuersätze und zur Finanzierung höherer Ausgaben für Forschung und Familie. In der Gesamtschau der finanziellen Implikationen des Regierungsprogramms kam Gert Keil zu der Einschätzung, dass „die neue Ehrlichkeit mit viel Scheinheiligkeit verbunden "101 sei, da je nach Institut eine Finanzierungslücke von 20 bis 40 Milliarden Euro bleibe. Der SPD-Finanzexperte Joachim Poß bezeichnete Merkel gar als „50-Milliarden-Risiko"102.

\subsection{Kontextualisierung der (Un-)Ehrlichkeit im Unionsprogramm}

Zur Kontextualisierung des Unionsprogramms ist erstens das Verhältnis von perzipierten Problemen und angebotenen Abhilfen zu charakterisieren. So wurde ein Auseinanderfallen der Beschreibung Deutschlands als Sanierungsfall und der präsentierten Lösungsvorschläge bemängelt. Deutschland werde als kaputtes Land beschrieben, eine Rosskur aber nur simuliert: „Diagnose und Therapie haben nicht die gleiche Affekttemperatur, sie passen einfach nicht zusammen."103

Zweitens war die „neue Ehrlichkeit“ der Union auch insofern problematisch, als sie reihenweise programmatische Festlegungen aus der Oppositionszeit außer Kraft setzte. Plötzlich wurde die Mehrwertsteuererhöhung, kurz zuvor noch als „Gift für die Konjunktur“ gegeißelt, offensiv vertreten. Die einst im Falle eines vorzeitigen Endes von Rot-Grün für den 1. Januar 2005 veranschlagte Einkommensteuersenkung entpuppte sich, kaum war die Situation eingetreten, als undurchführbar und wurde abgespeckt und verschoben. Von einer Abschaffung der Ökosteuer war keine Rede mehr. Bei der Körperschaftssteuer hatte die Union die Regierung beim „Job-Gipfel“ noch auf eine Senkung von 25 auf 19 Prozent festgelegt, beschränkte sich aber nun auf 22 Prozent. Kaum rückte die Regierungsübernahme in greifbare Nähe, erklärte sich „die potenzielle Kanzler-Partei für im Wesentlichen re-

99 Dorothea Siems, Rente mit 67, in: Die Welt vom 27. Juni 2005, S. 8.

100 Vgl. Spiegel Online vom 11. Juli 2005, http://www.spiegel.de/politik/deutschland/0,1518,364 773,00.html (Abruf am 12. August 2009).

101 Gert Keil, Ein abgemagerter Hamster mit prall gefüllten Backentaschen. Das Regierungsprogramm der CDU, in: Neue Gesellschaft. Frankfurter Hefte, Nr. 9/2005, S. 33 - 36, S. 34.

102 Zitiert nach Spiegel Online vom 12. Juli 2005, http://www.spiegel.de/politik/deutschland/0,1518,364843,00.html (Abruf am 12. August 2009).

103 Gert Keil, a.a.O. (Fn. 101), S. 33. Vgl. Jens Schneider, a.a.O. (Fn. 7). 
formunwillig und -unfähig" ${ }^{104}$. Durch die massive Abkehr von der bisherigen Programmatik desillusionierte sie nicht nur jene Wähler, die sich mit diesen Positionen identifiziert hatten, sondern gestand zugleich eine Vorwahl-Lüge ein, wodurch die Affirmation der „neuen Ehrlichkeit“ - durch die „alten“ Akteure - nicht glaubwürdiger wurde.

Drittens ist die Ehrlichkeit des Unionsprogramms in Beziehung zu setzen zu jener der Programme der anderen Parteien. Merkel sprach von einer „Relativwelt“, in der sie sich zu bewegen habe; wer angesichts der Konkurrenzlage populistischer Programme der SPD und der Linkspartei von ihr Sozialabbau-Thesen erwarte, verstehe wenig von Politik. ${ }^{105}$ Dass die potenzielle Unehrlichkeit der Gegner ein Problem eines ehrlichen Wahlkampfs darstellen kann, wurde bereits theoretisch diskutiert. Im konkreten Praxisfall vermochte die Union die gegenüber dem griffigen SPD-Plakatslogan „2 \% Merkelsteuer auf alles“ komplexere Realität nicht zu vermitteln, dass gerade sozial schwächere Gruppen ihr Einkommen vorwiegend für Waren und Dienstleistungen aufwenden, die entweder von der Mehrwertsteuer komplett befreit sind (wie etwa Mieten) oder dem reduzierten Mehrwertsteuersatz von sieben Prozent unterliegen (wie etwa Lebensmittel). Was das Wahlmanifest der SPD angeht, so kann man ihm zwar eine „Palliativ-Wirkung“106 nach den innerparteilich unbeliebten und elektoral desaströsen Hartz IV-Reformen zuschreiben oder es als „beeindruckendes Dokument programmatischer Erschöpfung "107 interpretieren, belegt etwa durch das Festhalten am „bewährten Einkommenssteuersystem“. Die geringfügigeren Änderungswünsche gegenüber dem tendenziell durch eine deutlich rosarotere Brille beurteilten Status quo rechtfertigen aber auch ein Weniger an Gegenfinanzierungselementen; die Deckungslücke war im Ergebnis mit etwa 20 Milliarden Euro nicht größer als die des Unionsprogramms. ${ }^{108}$ Dennoch scheint die Strategie der Union zumindest zum Teil aufgegangen zu sein: Die Versprechen des SPD-Manifests hielt nur ein Viertel der Wähler für glaubwürdig und nur ein Fünftel für finanzierbar; beim Unionsprogramm gingen zwar auch nur 27 Prozent von der Finanzierbarkeit aus, dafür bescheinigten ihm aber 42 Prozent Glaubwürdigkeit. ${ }^{109}$

Weitaus berechtigter war der Populismusvorwurf im Fall der Linkspartei. Sie kündigte zwar umfangreiche Mehreinnahmen zur Finanzierung ihrer Wahlversprechen an, angesichts der zahllosen in Aussicht gestellten sozialen Wohltaten verblieb aber eine Finanzierungslücke von mindestens 40 Milliarden Euro. ${ }^{110}$ Die Pläne der Grünen wiesen nach Angaben ihres eigenen Finanzpolitikers Oswald Metzger sogar über 70 Milliarden Euro Deckungslücke auf. ${ }^{111}$ Die FDP als Wunschkoalitionspartner der CDU/CSU plante Steuerentlastungen, die sie selbst mit 17 bis 19 Milliarden Euro bezifferte, Experten hingegen auf etwa

104 Marc Beise, Die Union - einfallslos und mutlos, in: SZ vom 28. Mai 2005, S. 25.

105 Vgl. Der Spiegel vom 11. Juli 2005, S. 39.

106 Martina Fietz, Gesiegt bis zum Umfallen, in: Cicero, Nr. 10/2005, S. 60 - 63, S. 61.

107 Berthold Löffler, a.a.O. (Fn. 33).

108 Vgl. Der Spiegel vom 1. August 2005, S. 31.

109 Vgl. Benjamin Hoff/ Steffen Twadowski, Schwarz-gelb und rot-grün verlieren die Wahl, S. 5 f., http://www.rosaluxemburgstiftung.de/fileadmin/rls_uploads/pdfs/Ergebnisse_BT-Wahl2005.pdf (Abruf am 15. Juli 2009).

110 Zweifelt man die Verfassungskonformität der kompletten Abschaffung des Ehegattensplittings und der Wiedereinführung der Vermögenssteuer an, klaffte sogar ein Loch von bis zu 150 Milliarden Euro, vgl. Der Spiegel vom 31. August 2005, S. 31.

111 Vgl. SZ vom 9. Juli 2005, S. 9. 
28 Milliarden schätzten. ${ }^{112}$ Zur Gegenfinanzierung wurde vage auf ein „bereits in den Haushaltsberatungen zum Bundeshaushalt 2005" aufgezeigtes Einsparvolumen von 12,5 Milliarden Euro verwiesen. Zusammen mit noch vageren „weiteren strukturellen Maßnahmen" ergebe sich eine Entlastung der öffentlichen Haushalte von circa 35 Milliarden Euro. ${ }^{13}$ Von einer Auflösung der Bundesagentur für Arbeit versprach sich die FDP Einsparungen von 15 Milliarden Euro; hierfür wäre wohl ein „Hinrichtungskommando für öffentlich Bedienstete"114 erforderlich gewesen. Trotz Unehrlichkeit in sämtlichen Wahlprogrammen war in allen auch - wenngleich in sehr unterschiedlicher Intensität - „eine Wende zum Weniger, das Austauschen von Wohltaten gegen Zumutungen"115 auszumachen. Die Union hat diese Wende wohl ungleich radikaler vollzogen als die übrigen Parteien, weniger in Bezug auf die Deckungslücke des Programms, sondern indem sie für nahezu alle gesellschaftlichen Gruppen Zumutungen bereithielt: für die abhängig Beschäftigten unter anderem Kürzung der Pendlerpauschale, Ende der Steuerfreiheit der Zuschläge für Sonn-, Feiertags- und Nachtarbeit, Ausweitung des Niedriglohnsektors, Verbetrieblichung der Lohnfindung und unbezahlte Mehrarbeit; für die Rentner, Studenten und Arbeitslosen Einkommenseinbußen durch die Erhöhung der Mehrwertsteuer. Darin unterschied sie sich nicht nur von SPD, Grünen und Linkspartei, die sich mit ihren Zumutungen überwiegend auf die „Reichen“ konzentrierten, sondern wich auch vom Pate stehenden Wahlkampf von Rüttgers ab, der sich auf Bergarbeiter und öffentlich Bedienstete beschränkt hatte. Dies könnte auch erklären, warum Rüttgers' Wahlkampf im Resultat besser funktionierte als der Bundestagswahlkampf der Union.

\section{4. (Un-)Ehrlichkeit in der Wahlkampfführung}

Den Ehrlichkeitsanspruch der Union konterkarierende Unehrlichkeiten finden sich nicht nur im Wahlprogramm, sondern auch in der Wahlkampfführung. Dabei lassen sich strategisch motivierte und situativ verursachte Unehrlichkeiten unterscheiden.

\subsection{Strategisch motivierte Unehrlichkeiten}

Die strategisch motivierten Unehrlichkeiten lassen sich analytisch auf die jeweils kausalen Strategien beziehen. Bestandteil des gouvernementalen Wahlkampfs war die bis zur Unglaubwürdigkeit überhöhte Behauptung eines im Vergleich zu Rot-Grün solideren Politikhandwerks einer unionsgeführten Regierung. Ebenso mit Erwartungen überfrachtet wurde die Verheißung des Durchregierens. Mit dem Beharren etlicher Unions-Ministerpräsiden-

112 Vgl. Deutsches Institut für Wirtschaftsforschung Berlin / Institut der deutschen Wirtschaft Köln, Beurteilung der Wahlprogramme zur Bundestagswahl 2005, Berlin / Köln 2005, S. 41, http://www.diw.de/deutsch/dasinstitut/info/2005/gutachten_zdf_2005.pdf (Abruf am 15. Juli 2009).

113 Vgl. FDP-Vorstand, Arbeit hat Vorfahrt. Deutschlandprogramm 2005, Berlin 2005, S. 7, http:// files.liberale.de/fdp-wahlprogramm.pdf (Abruf am 11. August 2009).

114 Vgl. Der Spiegel vom 1. August 2005, S. 31.

115 Karl-Rudolf Korte, Rückblick auf Ausnahme-Wahlen, in: Internationale Politik, 60. Jg. (2005), H. 10, S. $62-63$, S. 63. 
ten auf ihrem Anteil an der Mehrwertsteuererhöhung zulasten der Pläne der Bundespartei zur Senkung der Arbeitslosenversicherungsbeiträge war die Probe aufs Exempel bereits vor Verkündung des Regierungsprogramms öffentlichkeitswirksam gescheitert. ${ }^{116}$ Das rhetorische Beharren auf der Perspektive des Durchregierens nach dem verheerenden Praxistest kratzte das Ehrlichkeitsimage der Union an.

Eine Durchbrechung der Ehrlichkeitsstrategie im Dienste des Themenwahlkampfs stellte die Absage eines zweiten TV-Duells unter dem Vorwand von Zeitmangel dar. Die Vorgeschobenheit dieser Begründung wurde von fast drei Vierteln der Befragten erkannt ${ }^{117}$ und von Merkel schließlich mit ihrer Zusage für eine ZDF-Gesprächsrunde von Spitzenpolitikern aller Parteien faktisch selbst eingeräumt. Der Berater Stoibers im Bundestagswahlkampf 2002 Michael Spreng kritisierte, die Union hätte von Anfang an offensiv ein TV-Duell und eine „Elefantenrunde“ anbieten sollen. ${ }^{118}$ Zeitmangel diente auch als vorgeschobene Begründung für die Absage des traditionellen Besuchs deutscher Oppositionsführer im Weißen Haus. Anstelle der Kanzlerkandidatin fuhr der im Kompetenzteam für Außenpolitik zuständige Wolfgang Schäuble nach Washington. Motiv dürfte hier gewesen sein, Erinnerungen an Merkels vorbehaltlose Unterstützung der amerikanischen Entscheidung für den Irakkrieg zu vermeiden. ${ }^{119}$

Der Wirtschaftswahlkampf führte angesichts der Diskrepanz zwischen den steuerpolitischen Vorstellungen Kirchhofs und den Festlegungen im Unionsprogramm zu einem Lavieren Merkels und anderer Spitzenpolitiker der CDU/CSU zwischen diesen beiden Konzepten. Die Stellungnahmen oszillierten dabei zwischen Einebnen und Unterstreichen der Differenzen, zwischen Lobpreisungen des Reformers Kirchhof und Distanzierungen von seinen Positionen, wodurch deren Diskrepanz zu den offiziellen Unionsplänen erst vollständig virulent wurde. Es handelte sich dabei nicht um eine Kakophonie verschiedener innerparteilicher Strömungen, sondern um eine strategisch notwendige Doppelzüngigkeit: Einerseits musste zur Rechtfertigung von Kirchhofs Verbleib im Kompetenzteam und zur Aufrechterhaltung des durch ihn symbolisierten Reform-Impetus der Union der Eindruck erweckt werden, es gebe zwischen seinen Positionen und den Programmaussagen keinerlei Widersprüche und die im Programm skizzierte Steuerreform sei ein erster Schritt in Richtung Kirchhof. ${ }^{120}$ Andererseits war mit Blick auf Ängste in der Wählerschaft eine dosierte Distanzierung von ihm angezeigt, die in erster Linie die Form annahm, seine Pläne für eine Pauschalsteuer „in das milde Licht der Langfristigkeit zu setzen“121. So bezeichnete Merkel diese als „Vision“ und „Zukunftsmusik“, deren Umsetzung keinesfalls für die kommende Legislatur anstehe, und banalisierte sie zu unverbindlichem „Weiterdenken“, das ja nicht verboten sei. ${ }^{122}$ In der Stoiberschen Variante "Jeder darf seine Visionen haben" 123 klang gar generös-herablassende Nachsicht durch. Die Unions-Ministerpräsidenten meldeten große

116 Vgl. Bernd Ulrich, Alles auf eine Karte, in: Die Zeit vom 14. Juli 2005, S. 3. Vgl. auch SZ vom 11. Juli 2005, S. 3.

117 Vgl. Welt am Sonntag vom 7. August 2005, S.4.

118 Vgl. Michael H. Spreng, a.a.O. (Fn. 90), S. 10.

119 Vgl. SZ vom 29. Juli 2005, S. 4.

120 So Merkel, vgl. FAZ vom 23. August 2005, S. 1.

121 Bernd Ulrich, a.a.O. (Fn. 74).

$122 \mathrm{Vgl}$. FAZ vom 11. August, S. 4; SZ vom 30. August 2005, S. 6.

123 Zitiert nach SZ vom 30. August 2005, S. 6. 
Bedenken an, teilweise verklausuliert in pflichtschuldigen Lobesäußerungen. ${ }^{124}$ Dabei spielte wohl neben der Furcht um das Bestehen in künftigen Landtagswahlen eine Rolle, dass die Pauschalsteuer im ersten Jahr Einnahmeverluste von mehr als 40 Milliarden Euro bedeutet hätte, von denen die Länder 42,5 Prozent hätten tragen müssen. ${ }^{125}$ Auch andere Spitzenpolitiker der Union distanzierten sich. Damit spielten sie der SPD in die Hände, da ihr Abrücken als Bestätigung von deren Vorwürfen interpretiert werden konnte. ${ }^{126} \mathrm{Zu}-$ gleich dürften die Distanzierungen Merkels Autorität in Zweifel gezogen und Anhänger von Radikalreformen zur FDP getrieben haben. Guido Westerwelle lockte diese mit der Ankündigung, die FDP werde dafür sorgen, dass Kirchhofs Vorstellungen auch Position einer schwarz-gelben Koalition würden. ${ }^{127}$ Gleichzeitig unterlief er so die Bemühungen der Union, die Pauschalsteuer als eine in der folgenden Wahlperiode auf keinen Fall anstehende Maßnahme zu präsentieren. Ohnehin dürften sich die Wähler angesichts der Erschöpfung des rot-grünen Projekts und der üblichen Verweildauer von Bundesregierungen auf eine schwarz-gelbe Koalition über mindestens acht Jahre eingerichtet haben, so dass die Ankündigung, die Pauschalsteuer werde erst 2009 ein Thema, wohl weniger beruhigende Wirkung entfaltete, als dies von Unionsseite intendiert gewesen war. Das die Distanzierung von Kirchhof begleitende Verwirrspiel um eine Tandemlösung mit Friedrich Merz war wenig hilfreich. Stoibers öffentlich zur Schau gestellte Unentschlossenheit hinsichtlich seiner Rolle in einer Regierung Merkel untergrub ebenfalls den Ehrlichkeitskurs: „Die Menschen bekamen den Eindruck, manch Wichtiges wolle die Union vor der Wahl doch nicht verraten." 128

Zugunsten des Lagerwahlkampfes wurden im Abschlusspapier des Wechselgipfels sämtliche strittigen Themen ausgeklammert, wodurch sich der Gipfel als kaum verhohlene reine „PR-Show für eine neue Regierung“129 entpuppte. Das nur längst bekannte und hinreichend vage Schnittmengen beschreibende Papier war von den Generalsekretären im Vorfeld ausgearbeitet worden und weder Ergebnis noch Hauptgegenstand der knapp einstündigen Unterredung der Spitzenpolitiker von Union und FDP. ${ }^{130}$ Die sich gern über die Darstellungskünste des Medienkanzlers mokierende Kandidatin erwies sich selbst als „vollends kontrollierte Selbstdarstellerin“131. Zu ihrem Ehrlichkeitsimage passten auch die von den Aktivisten des „teAM Zukunft“ bei jedem Auftritt aufgesetzt enthusiastisch geschwenkten Pappschilder nicht wirklich. ${ }^{132}$ Die Inszenierung als Graswurzelbewegung passte zwar abstrakt besser zur Ehrlichkeitsstrategie als das Herausstellen des Spin Doctors Spreng 2002, wirkte durch ihre Anleihen beim amerikanischen Wahlkampfstil jedoch ambivalent.

Den Erfordernissen des Lager-, aber auch des Regierungswahlkampfs ist eine weitere Unehrlichkeit geschuldet: das kategorische Ausschließen einer großen Koalition. Je weiter Schwarz-Gelb in den Umfragen absackte, desto mehr musste diese Absage mit der War-

124 Vgl. Beispiele in: Der Spiegel vom 29. August 2005, S. 42.

125 So eine Berechnung aus dem Stuttgarter Finanzministerium, vgl. FAZ vom 31. August 2005, S. 4.

126 Vgl. Michael H. Spreng, a.a.O. (Fn. 90), S. 11.

127 Vgl. FAZ vom 22. August 2005, S. 11.

128 Stephan Eisel, a.a.O. (Fn. 4), S. 51.

129 Jens Schneider, a.a.O. (Fn. 84).

130 Vgl. Die Welt vom 2. September 2005, S. 6.

131 Vgl. Jens Schneider, a.a.O. (Fn. 84).

132 Vgl. Stephan Eisel, a.a.O. (Fn. 4), S. 48. 
nung vor einem rot-rot-grünen Linksbündnis einhergehen. Um diese Gefahr trotz entgegenstehender strategischer Interessen und persönlicher Animositäten der Parteispitzen sowie fehlender Unterstützung der Anhängerschaften ${ }^{133}$ plausibel zu machen, wurde ein Menetekel-Charakter der rot-roten Landeskoalitionen in Berlin und Mecklenburg-Vorpommern heraufbeschworen. ${ }^{134}$ Beteuerungen Schröders, er werde nicht mit der Linkspartei koalieren, wurden dabei durchaus perfide als glaubhaft, aber irrelevant eingestuft, da dieser dann in der SPD nicht mehr das Sagen haben werde. ${ }^{135}$ Dabei konterkarierte allerdings Merkel ihr eigenes Argumentationsmuster, indem sie eine große Koalition selbst als letztes Mittel zur Verhinderung des als verheerend beschriebenen Linksbündnisses ablehnte. ${ }^{136}$ Das apodiktische Ausschließen einer großen Koalition auch für den Fall, dass es die einzige Möglichkeit wäre zu regieren ${ }^{137}$, beschädigte angesichts der Umfragedaten die Glaubwürdigkeit der Union. Dieser Effekt wurde verstärkt durch die - freilich angesichts der Prognosen wahltaktisch gebotene - geschmeidigere Haltung der SPD. ${ }^{138}$

Vor die Alternative gestellt, ihren Ehrlichkeitsanspruch in der operativen Wahlkampfführung einzulösen oder ihre anderen Strategien zu behaupten, entschied sich die Union regelmäßig für letztere Option. Wie bereits dargelegt ist Ehrlichkeit aber eine moralische Kategorie mit dem damit einhergehenden Absolutheitsanspruch und schließt, nimmt man sie ernst, eine solche Güterabwägung per Definition aus.

\subsection{Situativ verursachte Unehrlichkeiten}

Weniger strategischer Natur als vielmehr situativ auftretenden Ereignissen geschuldet waren weitere unehrliche Brechungen im Ehrlichkeitswahlkampf der Union.

Hatte Merkel in Abkehr von Schröders gebrochenem Versprechen einer Halbierung der Arbeitslosigkeit konkrete Festlegungen zu Erfolgen am Arbeitsmarkt vermieden, da sie derartige Prognosen redlicherweise nicht treffen könne, setzte Stoiber nur wenige Tage später die österreichische Arbeitslosenquote in Höhe von vier Prozent als Marke. ${ }^{139}$ Die Dementis und Distanzierungen blieben halbherzig.

Einiges Aufsehen erregte der Umgang mit dem Brutto-Netto-Verwechsler der Kandidatin in der ARD-Sendung „Bericht aus Berlin“. Dort hatte Merkel gesagt, die Reduzierung der Lohnzusatzkosten bedeute eine Absenkung der Bruttolöhne um ein Prozent. ${ }^{140}$ Auf der CDU-Homepage wurde das „Wortlaut“-Interview veröffentlicht, wobei die Absenkung der

133 Vgl. Hans Rattinger / Zoltan Juhasz, a.a.O. (Fn. 26), S. 16.

134 Vgl. das Interview mit Merkel in: SZ vom 20. Juli 2005, S. 7.

135 So Stoiber, vgl. Stern vom 7. Juli 2005, S. 33.

136 Vgl. FAZ vom 24. Juni 2005, S. 3.

137 Vgl. SZ vom 20. Juli 2005, S. 7.

138 Vgl. FAZ vom 22. August 2005, S. 1.

139 Vgl. Interview in: Bild am Sonntag vom 17. Juli 2005, S. 7.

$140 \mathrm{Merkel}$ hatte sich dagegen ausgesprochen, die private Zusatzvorsorge mit der Riester-Rente zur Pflicht zu machen, und dann ausgeführt: „Der interessante Effekt, den wir jetzt haben, ist ja, dass für alle Arbeitnehmerinnen und Arbeitnehmer - und für die gilt ja die Riester-Rente - die Brutto-Löhne um ein Prozent sinken, wenn wir die Lohnzusatzkosten senken. Und das gibt auch wieder Spielraum, um zum Beispiel für die eigene Altersvorsorge etwas zu tun." Zitiert nach Nico Fried, Angela Scharping, in: SZ vom 3. August 2005, S. 1. Vgl. Die Welt vom 4. August 2005, S. 8. 
Bruttolöhne stillschweigend zunächst in eine Steigerung der Bruttolöhne und schließlich in eine Steigerung der Nettolöhne verwandelt wurde. ${ }^{141}$ Die angesichts der behaupteten Wirtschaftskompetenz und handwerklichen Solidität von Kandidatin und Partei peinliche Verwechslung erzeugte ein hämisches Medienecho. ${ }^{142}$ Ein richtiges Problem wurde sie aber erst durch den linkischen Vertuschungsversuch. ${ }^{143}$ Eventuell hätte Merkel durch das Eingeständnis des Fehlers ihr Ansehen bei den Wählern sogar stärken können. ${ }^{144}$ So aber beschädigte die Union ihre Ehrlichkeitsstrategie nachhaltig, ohne verhindern zu können, dass ihr Versprechen handwerklicher Solidität zunehmend zur Schimäre wurde.

Der Ehrlichkeitsstrategie abträglich war zudem die von der SPD zur „Giftliste“ stilisierte Aufstellung der nach Ansicht Kirchhofs streichenswerten Steuervergünstigungen, deren ersten Entwurf von 2001 seine Mitarbeiter von der Internet-Seite der Universität Karlsruhe entfernten - angeblich schon ein halbes Jahr zuvor. ${ }^{145}$ Nach ihrer Auskunft lag die Liste bei der Union, was die CDU-Bundesgeschäftsstelle bestritt. Die Forderung einer Veröffentlichung der Streichliste durch Rot-Grün konterte die Union mit dem Vorwurf, die Regierung halte ihrerseits eine Sparliste im präzedenzlosen Volumen von 30 Milliarden Euro zurück und plane im Rahmen dieses wiederholten Wahlbetrugs nach 2002 eine Erhöhung der Mehrwertsteuer inklusive Streichung des reduzierten Satzes zum Beispiel für Lebensmittel. ${ }^{146}$ Es konnte hierdurch der Eindruck entstehen, die Union versuche „die letzten Tage des Wahlkampfs mit erfundenen und bestellten Geschichten zu überstehen"147.

Auch das Last-Minute-Wahlkampfmanöver in Sachen Ökosteuer dürfte wenig Stimmen eingebracht, aber viel Glaubwürdigkeit gekostet haben. Zwei Wochen vor der Wahl revitalisierte Merkel die im Programm jäh kassierte langjährige Forderung unter Hinweis auf die stark gestiegenen Benzinpreise. Da Rot-Grün nicht die gesamte Ökosteuer in die Finanzierung der Rente gegeben habe, sondern drei Cent pro Liter in den Haushalt solle nach einem Wahlsieg eine Senkung der Ökosteuer um diesen Betrag überprüft werden. ${ }^{148}$ Diese Verlautbarung erschien nicht nur aufgrund ihrer Kurzfristigkeit und Vagheit zweifelhaft; sie widersprach auch der Schilderung der angespannten Lage sowohl der Rentenkasse als auch des Haushalts im Regierungsprogramm, das für keinen der beiden Bereiche einen auch nur annähernd adäquaten Maßnahmenkatalog aufzeigte.

141 Vgl. Die Welt vom 4. August 2005, S. 8.

142 Vgl. zum Beispiel Spiegel Online vom 3. August 2005, http://www.spiegel.de/politik/deutschland/0,1518,368085,00.html (Abruf am 12. August 2009); SZ vom 3. August 2005, S. 1; taz vom 4. August 2005, S. 2.

143 Vgl. Michael H. Spreng, a.a.O. (Fn. 90), S. 10. Zudem erschien der Brutto-Netto-Verwechsler durch einen Autorisierungsfehler ein paar Tage später in einem weiteren Interview, vgl. SZ vom 4. August 2005, S. 5.

144 Dieser Ansicht ist Peter Dausend, Merkel, in: Die Welt vom 4. August 2005, S. 8.

145 Vgl. Der Spiegel vom 12. September 2005, S. 35.

146 Vgl. FAZ vom 14. September 2005, S. 4.

147 Nina Bovensiepen, Merkel-Minus gegen Eichel-Gift, in: SZ vom 14. September, S. 20. Laut Bovensiepen spricht einiges dafür, dass CDU-nahe Mitarbeiter im Finanzministerium bei Anfertigung und Veröffentlichung der Sparliste nachhalfen, die ursprünglich in erster Linie Verkehrsprojekte enthielt und Einsparmöglichkeiten von lediglich 1,8 Milliarden Euro aufzeigte. Die Zahl 30 Milliarden Euro brachte erst Volker Kauder unter Berufung auf eigene Gespräche mit Beamten aus dem Finanzministerium ins Spiel, vgl. FR vom 15. September 2005, S. 4.

148 Vgl. Die Welt vom 3. September 2005, S. 4. 
Insgesamt konnte die CDU/CSU ihren hoch gesteckten Ehrlichkeitsansprüchen in der operativen Wahlkampfführung nicht durchgehend genügen. Inkonsequentes Verhalten war in diesem Bereich von der Öffentlichkeit leichter wahrnehmbar und verursachte somit größere Schäden am Ehrlichkeitsimage als Unklarheiten im Wahlprogramm.

\subsection{Die Wirkung der Ehrlichkeitsstrategie}

Viele Beobachter zogen angesichts des Ausgangs der Bundestagswahl 2005 ohne vorhergehende detaillierte Analyse den Schluss, Merkel sei „zu ehrlich“ gewesen: Man könne mit der immer wieder geforderten Aufrichtigkeit offenbar doch keine Wahlen gewinnen. ${ }^{149}$

Sicherlich rächte es sich, dass die Union für so gut wie jede Wählergruppe Zumutungen bereithielt und damit von Rüttgers' erfolgreichem Wahlkampfkonzept abgerückt war. Resignative Erklärungsmuster wie „Die Wähler wollen nun mal belogen werden“ scheinen naheliegend, greifen jedoch nur zu einem Teil. Wie gesehen war die Union allenfalls „relativ ehrlich“, von der absoluten Ehrlichkeit, die sie vehement für sich reklamierte, aber weit entfernt. ${ }^{150}$ Dies dürfte sich in puncto Unionsprogramm weniger gerächt haben, da die wenigsten Bürger dessen angeblich ausgeglichene Bilanz überprüft haben werden. Insofern ist der Plan, Vagheiten und Unehrlichkeiten hinter plakativen Zumutungen zu verstecken, wahrscheinlich weitgehend aufgegangen. Vor allem auf der Ebene der Wahlkampfführung hat die Union aber mit weit offenkundigeren und teilweise unnötigen Unehrlichkeiten ihre Ehrlichkeitsstrategie öffentlichkeitswirksam unterminiert und sich damit selbst um die Früchte der Entbehrungen gebracht, die sie in einem an Versprechen armen und an erklärungsbedürftigen Zumutungen reichen Wahlkampf auf sich genommen hatte. Am Ende des Wahlkampfs hatte Schröder seinen Glaubwürdigkeitsrückstand von 17 Prozentpunkten auf Merkel bis auf drei Punkte aufgeholt, während diese ihren höchsten Wert direkt nach ihrer Nominierung verbuchen konnte, als die Union programmatisch nur ihre von Aufrichtigkeit ungetrübten Forderungen der Oppositionsjahre aufgeboten hatte. ${ }^{151}$ Der Union gelang es dergestalt nicht, ihre Sicht der Dinge zur alternativlosen „Wirklichkeit“ zu stilisieren, der man sich zu unterwerfen habe; die Wähler behielten somit die Wahl zwischen verschiedenen Maßnahmenkatalogen, unter denen derjenige der Union vielen nicht zusagte.

Im Ergebnis vermochte die Union zwar ihren Ehrlichkeitsanspruch deutlich zu machen und damit stellenweise die öffentliche Diskussion in ihrem Sinne zu beeinflussen. Dieser Anspruch wurde aber weder von der Union voll ausgefüllt noch von den Wählern in einem Maße honoriert, das die mit dieser Strategie einhergehenden Einschränkungen aufwiegen konnte. Der Ehrlichkeitswahlkampf schadete der Union mithin mehr, als er ihr nutzte.

149 Vgl. zum Beispiel Frank Priess, Ein Wahlkampf der besonderen Art, in: Die Politische Meinung, Nr. 10/2005, S. 10.

150 Der jetzige Kanzleramtschef Thomas de Maizière merkte als einziger Spitzenpolitiker der Union selbstkritisch an, die Union sei, wenn auch immerhin ehrlicher als die anderen Parteien, vielleicht noch nicht ehrlich genug gewesen, vgl. Spiegel Online vom 9. November 2005, http:// www.spiegel.de/kultur/gesellschaft/0,1518,383993,00.html (Abruf am 12. August 2009).

151 Vgl. Infratest dimap, ARD-DeutschlandTrend Juni 2005, S. 15 und September 2005 II, S. 6. 


\section{Wie stehen die Chancen für Ehrlichkeitsstrategien in der Zukunft?}

Bei der Wahlaussprache der Union am 6. Dezember 2005 wurde der Ehrlichkeitswahlkampf außer dem Wirtschaftswahlkampf als einzige der sechs hier thematisierten Strategien diskutiert. Das Fazit der Union blieb dabei eigentümlich gespalten: In Hintergrundgesprächen dominierte das Erklärungsmuster „Der Ehrliche ist der Dumme“. Von Politikern der zweiten Reihe ließ sich dieser Tenor auch öffentlich vernehmen. ${ }^{152}$ Das Spitzenpersonal der Union verteidigte hingegen die Ehrlichkeitsstrategie als vollkommen richtig. Wenn man damit nicht so erfolgreich gewesen sei wie erhofft, habe dies daran gelegen, dass die Menschen das Vertrauen in die Gestaltungsfähigkeit der Politik verloren hätten, die bei ihnen zu oft Erwartungen geweckt und dann enttäuscht habe. ${ }^{153}$ Die scharfe Trennlinie zwischen den Äußerungen von Akteuren der zweiten und der ersten Reihe verweist auf die strategische Natur der Äußerungen der Spitzenpolitiker: Eine formelle Distanzierung vom Ehrlichkeitswahlkampf hätte für Ministerpräsidenten wie Wulff und Koch, die 2008 ihre Ämter zu verteidigen hatten, eine schwere Hypothek dargestellt. Nicht einmal nach Abschluss des Wahlkampfs konnte „die erhabene Tiara"154 abgelegt werden.

Die offiziellen Bekenntnisse zum Ehrlichkeitskurs in künftigen Wahlkampagnen sind also eine Sache, die tatsächliche Anwendung dieses Rezepts eine andere. Wird die Ehrlichkeitsstrategie nach den schlechten Erfahrungen der Union im Wahlkampf 2005 also still beerdigt werden ${ }^{155}$, oder sind die Chancen zur Mobilisierung von Mehrheiten für unpopuläre Entscheidungen gestiegen? ${ }^{156}$ Ein Verzicht auf unrealistische Versprechungen kann zumindest für jene Parteien, die eine Regierungsverantwortung anstreben und folglich vom Wähler auch am Maßstab der Regierungsfähigkeit gemessen werden, sinnvoll sein, weil sie auf diese Weise ihre Glaubwürdigkeit stärken und einer Wiederwahl abträglichen Frustrationen vorbeugen können. ${ }^{157}$ Es ist aber anzunehmen, dass die Parteien sich auf einige wenige Zumutungen für möglichst klar umrissene Wählersegmente beschränken und Ehrlichkeit nicht mehr zum Leitthema ausrufen werden. So könnten sie verhindern, beim Wähler übersteigerte Ansprüche an ihre Ehrlichkeit zu generieren, die aufgrund des strategischen Charakters von Wahlkämpfen in der Kampagnenführung kaum einzulösen sind.

152 Vgl. FAZ vom 20. September 2005, S. 53; SZ vom 24. September 2005, S. 3; Welt am Sonntag vom 23. Oktober 2005, S. 6; Die Welt vom 6. Dezember 2005, S. 2; Der Spiegel vom 1. Oktober 2005, S. 29.

153 Vgl. FAZ vom 2. Dezember 2005, S. 13 und vom 6. Dezember 2005, S. 1.

154 Marcel Proust, a.a.O. (Fn. 65), S. 265.

155 Vgl. Ansgar Graw, Merkels Spagat, in: Die Welt vom 6. Dezember 2005, S. 8; Rainer Hank I Winand von Petersdorf, Die Angst vor der eigenen Courage, in: Frankfurter Allgemeine Sonntagszeitung vom 25. September 2005, S. 35.

156 Vgl. Karl-Rudolf Korte, Was entschied die Bundestagswahl 2005?, a.a.O. (Fn. 54), S. 17.

157 Theoretisch sollte dieser Aussage desto mehr Gültigkeit zukommen, je angespannter die wirtschaftliche Lage ist. Die aktuelle Finanzkrise scheint mit dem Dogma der Haushaltsdisziplin auch diese Logik vorübergehend außer Kraft zu setzen, weil kostenintensive Wahlversprechen von den Parteien als konjunkturfördernde Maßnahme ausgegeben werden können. 